\title{
Supersymmetric solutions from $N=6$ gauged supergravity
}

\author{
Parinya Karndumri॰* and Jakkapat Seeyangnok ${ }^{\dagger}$ \\ String Theory and Supergravity Group, Department of Physics, Faculty of Science, \\ Chulalongkorn University, 254 Phayathai Road, Pathumwan, Bangkok 10330, Thailand
}

(Received 9 January 2021; accepted 4 March 2021; published 26 March 2021)

\begin{abstract}
We study supersymmetric solutions in four-dimensional $N=6$ gauged supergravity with the $S O(6)$ gauge group. There is a unique $N=6$ supersymmetric $\mathrm{AdS}_{4}$ vacuum with $S O(6)$ symmetry dual to an $N=6$ superconformal field theory (SCFT) in three dimensions. We find a number of domain walls interpolating between this $\mathrm{AdS}_{4}$ vacuum and singular geometries in the IR with $S O(2) \times S O(4), U(3)$, $S O(3)$, and $S O(2) \times S O(2) \times S O(2)$ symmetries. The $S O(3)$ case admits $N=6$ or $N=2$ solutions depending on whether the pseudoscalars are present or not. On the other hand, all the remaining solutions preserve $N=6$ supersymmetry. These solutions describe renormalization-group (RG) flows from the $N=6$ SCFT to nonconformal field theories driven by mass deformations. In particular, the $S O(2) \times$ $S O(4)$ solution is in agreement with the previously known mass deformations of the dual $N=6 \mathrm{SCFT}$. We also give a supersymmetric Janus solution with $S O(2) \times S O(4)$ symmetry, describing two-dimensional conformal defects in the $N=6$ SCFT with unbroken $N=(4,2)$ supersymmetry. Finally, we find an $N=2$ supersymmetric $\mathrm{AdS}_{2} \times H^{2}$ solution with $S O(2) \times S O(4)$ symmetry and the corresponding domain wall interpolating between this fixed point and the $\mathrm{AdS}_{4}$ vacuum. The solution describes an $\mathrm{AdS}_{4}$ black hole with a magnetic charge and is dual to a twisted compactification of the $N=6$ SCFT on a hyperbolic space $H^{2}$. We also give a domain wall interpolating between a locally supersymmetric $\mathrm{AdS}_{4}$ and a curved domain wall with $S O(2) \times S O(2) \times S O(2)$ symmetry dual to an RG flow across dimensions from the $N=6$ SCFT to a supersymmetric quantum mechanics.
\end{abstract}

DOI: $10.1103 /$ PhysRevD.103.066023

\section{INTRODUCTION}

Supersymmetric solutions of gauged supergravities in various space-time dimensions play an important role in string and M theory. In the AdS/CFT correspondence [1-3], these solutions provide holographic descriptions of strongly coupled systems such as (non)conformal field theories, conformal defects, AdS black holes, and condensed matter physics. In many cases, solutions of lowerdimensional gauged supergravities can be uplifted to ten or 11 dimensions via consistent truncations resulting in complete AdS/CFT dualities in the context of string and $M$ theory.

In this paper, we are interested in supersymmetric solutions of four-dimensional $N=6$ gauged supergravity with the $S O(6)$ gauge group; see [4] for all timelike supersymmetric solutions in ungauged $N=6$ supergravity.

\footnotetext{
*parinya.ka@hotmail.com

jakkapatjtp@gmail.com
}

Published by the American Physical Society under the terms of the Creative Commons Attribution 4.0 International license. Further distribution of this work must maintain attribution to the author(s) and the published article's title, journal citation, and DOI. Funded by SCOAP ${ }^{3}$.
This has been constructed in Ref. [5] in the embedding tensor formalism obtained from truncating the maximal $N=8$ gauged supergravity [6]; see also [7-9]. The $N=6$ gauged supergravity has been shown to admit a unique $N=6$ supersymmetric $\mathrm{AdS}_{4}$ vacuum, with the full $S O(6)$ symmetry unbroken, dual to an $N=6$ superconformal field theory (SCFT) in three dimensions. The uniqueness of the $N=6 \mathrm{AdS}_{4}$ vacua has also been shown in a recent result on supersymmetric AdS vacua [10]. It has been pointed out in Ref. [5] that this $\mathrm{AdS}_{4}$ fixed point describes a truncation of type IIA theory on $C P^{3}$, so the $\mathrm{AdS}_{4}$ vacuum can be uplifted to $\mathrm{AdS}_{4} \times C P^{3}$ geometry in type IIA theory. This truncation has been studied long ago in Ref. [11], in which the full mass spectrum has also been given; for more recent studies, see, for example, [12-15].

The very first example of the dual $N=6$ SCFT from type IIA theory has been given in Ref. [16]. In general, SCFTs in three dimensions take the form of Chern-Simonsmatter (CSM) theories, since the usual gauge theories with Yang-Mills gauge kinetic terms are not conformal. A number of these SCFTs with different numbers of supersymmetries have already been constructed; see [17-36] for an incomplete list. These SCFTs arise as world-volume theories of M2-branes on various transverse spaces and play an important role in understanding the dynamics of 
M2-branes. Supersymmetric solutions of four-dimensional gauged supergravities are expected to be very useful for their holographic descriptions, at least in the large- $N$ limit.

Various types of supersymmetric solutions from gauged supergravities have been considered and given interpretations in terms of the corresponding dual field theories. We will study these solutions in $N=6$ gauged supergravity beginning with supersymmetric domain walls interpolating between the $N=6$ supersymmetric $\mathrm{AdS}_{4}$ vacuum and singular geometries. The solutions describe holographic renormalization-group (RG) flows from the dual $N=6$ SCFT in the UV to nonconformal phases in the IR arising from mass deformations of the UV $N=6$ SCFT. Similar solutions have been extensively studied in $N=8$ and $N=2$ gauged supergravities; see, for example, [37-45]. Solutions in gauged supergravities with $N=3,4,5$ supersymmetries have also been considered recently in Refs. [46-51]. This work could hopefully fill the existing gap by providing a number of supersymmetric solutions in $N=6$ gauged supergravity.

We will also find Janus solutions in the form of $\mathrm{AdS}_{3^{-}}$ sliced domain walls interpolating between asymptotically $\mathrm{AdS}_{4}$ spaces. These are holographically dual to twodimensional conformal defects within the $N=6$ SCFT and break the superconformal symmetry in the threedimensional bulk to a smaller one on the two-dimensional surfaces. Solutions of this type in other four-dimensional gauged supergravities have previously been studied in Refs. [48,49,51-55]. Finally, we will look for $\mathrm{AdS}_{2} \times \Sigma^{2}$ geometries with $\Sigma^{2}$ being a Riemann surface together with solutions interpolating between these backgrounds and the supersymmetric $\mathrm{AdS}_{4}$ vacuum. The solutions describe supersymmetric black holes in asymptotically $\mathrm{AdS}_{4}$ space, and a number of these solutions have already been studied in other gauged supergravities in Refs. [51,56-66]. In the dual field theory, the solutions describe RG flows from the $N=6$ SCFT to superconformal quantum mechanics in the IR which play a prominent role in microscopic computation of black hole entropy in asymptotically $\mathrm{AdS}_{4}$ spaces; see, for example, [67-69]. In this context, the superconformal quantum mechanics, or one-dimensional SCFT, is obtained from the $N=6$ SCFT via twisted compactifications on $\Sigma^{2}$.

Four-dimensional $N=6$ gauged supergravity has $S O^{*}(12)$ global symmetry with the maximal compact subgroup $U(6) \sim S U(6) \times U(1)$. There are 30 scalars encoded in the $S O^{*}(12) / U(6)$ coset manifold. The $S O(6)$ gauging of this supergravity can be obtained from a consistent truncation of the maximal $N=8$ gauged supergravity with the $S O(8)$ gauge group. The latter is, in turn, a consistent truncation of 11-dimensional supergravity on $S^{7}$ [70-75]. The $N=6$ gauged supergravity with the $S O(6)$ gauge group can accordingly be uplifted to 11 dimensions via a series of consistent truncations. On the other hand, the $S O(6), N=6$ gauged supergravity is a consistent truncation of type IIA theory on $C P^{3}$. Therefore, all the solutions given here have known higher-dimensional origins and can be embedded in ten- or 11-dimensional supergravities. The scalar potential of the $N=6$ gauged supergravity has been analyzed for a long time in Ref. [76]. More recently, this gauged supergravity has been rewritten in a more general setting of the embedding tensor formalism in Ref. [5], in which the fermion-shift matrices and the scalar potential have been given by truncating the $N=8$ theory. In this paper, we first complete the task by extending the truncation to all terms in the bosonic Lagrangian and fermionic supersymmetry transformations. Both of these are, of course, a relevant part in the present analysis.

The paper is organized as follows. In Sec. II, we review four-dimensional $N=6$ gauged supergravity with $S O(6)$ gauge group in the embedding tensor formalism. In Sec. III, we study supersymmetric domain wall solutions describing RG flows in the dual $N=6 \mathrm{SCFT}$ to nonconformal phases in the IR. We then turn to supersymmetric Janus solutions in Sec. IV and finally look for possible supersymmetric $\mathrm{AdS}_{2} \times \Sigma^{2}$ solutions together with flow solutions interpolating between the $\mathrm{AdS}_{4}$ vacuum and these geometries in Sec. V. Conclusions and comments are given in Sec. VI.

\section{II. $N=6$ GAUGED SUPERGRAVITY WITH $S O(6)$ GAUGE GROUP}

We first give a review of $N=6$ gauged supergravity in the embedding tensor formalism as described in Ref. [5]. We will follow most of the convention in Ref. [5] but with a mostly plus signature for the space-time metric. The only supermultiplet in $N=6$ supersymmetry is the gravity multiplet with the field content

$$
\left(e_{\mu}^{\hat{\mu}}, \psi_{\mu A}, A_{\mu}^{A B}, A_{\mu}^{0}, \chi_{A B C}, \chi_{A}, \phi_{A B}\right) .
$$

The component fields correspond to the graviton $e_{\mu}^{\hat{\mu}}$, six gravitini $\psi_{\mu A}, 16$ vectors $A_{\mu}^{A B}=-A_{\mu}^{B A}$ and $A_{\mu}^{0}$, and 26 spin$\frac{1}{2}$ fields $\chi_{A B C}=\chi_{[A B C]}$ and $\chi_{A}$ together with 15 complex scalars $\phi_{A B}=-\phi_{B A}$. Real and imaginary parts of $\phi_{A B}$ are usually called scalars and pseudoscalars, respectively.

In this work, space-time and tangent space indices are denoted by $\mu, \nu, \ldots=0,1,2,3$ and $\hat{\mu}, \hat{\nu}, \ldots=0,1,2,3$, respectively. Indices $A, B, \ldots=1,2, \ldots, 6$ correspond to the fundamental representation of $S U(6)$ which is, in turn, a subgroup of the R-symmetry $U(6) \sim S U(6) \times U(1)$. The 30 real scalars within $\phi_{A B}$ are coordinates of the scalar manifold $S O^{*}(12) / U(6)$ and can be described by the coset representative in representation 32 of $S O^{*}(12)$ of the form

$$
\mathcal{V}_{M^{\underline{M}}}=\mathcal{A}^{\dagger} e^{Y}
$$

with the Cayley matrix 


$$
\mathcal{A}=\frac{1}{\sqrt{2}}\left(\begin{array}{cc}
\mathbb{I}_{16} & i \mathbb{I}_{16} \\
\mathbb{I}_{16} & -i \mathbb{I}_{16}
\end{array}\right)
$$

and

$$
Y=\left(\begin{array}{cccc}
0 & 0_{1 \times 15} & 0 & \phi_{C D} \\
0_{15 \times 1} & 0_{15 \times 15} & \phi_{A B} & \frac{1}{2} \epsilon_{A B C D E F} \bar{\phi}^{E F} \phi_{C D} \\
0 & \bar{\phi}^{C D} & 0 & 0_{1 \times 15} \\
\bar{\phi}^{A B} & \frac{1}{2} \epsilon^{A B C D E F} \phi_{E F} & 0_{15 \times 1} & 0_{15 \times 15}
\end{array}\right) .
$$

We also note that $\bar{\phi}^{A B}=\left(\phi_{A B}\right)^{*}$.

In subsequent analysis, it is useful to define $16 \times 16$ submatrices of $\mathcal{V}_{M^{\underline{M}}}$ by the following identification:

$$
\mathcal{V}_{M^{\underline{M}}}=\left(\begin{array}{ll}
\bar{h}_{\Lambda} \underline{\Lambda} & h_{\Lambda \underline{\Lambda}} \\
\bar{f}^{\Lambda} & f^{\Lambda}{ }^{\Lambda}
\end{array}\right)
$$

in which $\mathbf{f}, \mathbf{h}, \overline{\mathbf{f}}$, and $\overline{\mathbf{h}}$ satisfy the relations

$$
\left(\mathbf{f f}^{\dagger}\right)^{T}=\mathbf{f f}^{\dagger}, \quad\left(\mathbf{h h}^{\dagger}\right)^{T}=\mathbf{h} \mathbf{h}^{\dagger}, \quad \mathbf{f} \mathbf{h}^{\dagger}-\overline{\mathbf{f}} \mathbf{h}^{T}=i \mathbb{I}_{16},
$$

$\mathbf{f}^{\dagger} \mathbf{h}-\mathbf{h}^{\dagger} \mathbf{f}=-i \mathbb{I}_{16}, \quad \mathbf{f}^{T} \mathbf{h}-\mathbf{h}^{T} \mathbf{f}=0$.

The inverse of $\mathcal{V}_{M} \underline{\underline{M}}$ can accordingly be written in terms of $\mathbf{f}$ and $\mathbf{h}$ as

$$
\mathcal{V}_{\underline{M}}{ }^{M}=\left(\begin{array}{cc}
-i f^{\Lambda} \underline{\Lambda} & i h_{\Lambda} \underline{\Lambda} \\
i \bar{f}^{\Lambda} \underline{\Lambda} & -i \bar{h}_{\Lambda} \underline{\Lambda}
\end{array}\right) .
$$

The 16 electric gauge fields $A^{A B}$ and $A^{0}$ combine into $A^{\Lambda}=\left(A^{0}, A^{A B}\right)$. Together with the magnetic dual $A_{\Lambda}$, the gauge fields transform as the $\mathbf{3 2}$ representation of $S O^{*}(12)$ :

$$
A^{M}=\left(A^{\Lambda}, A_{\Lambda}\right) .
$$

Gaugings are efficiently described by the embedding tensor formalism in which the corresponding gauge generators are defined as

$$
X_{M}=\theta_{M}{ }^{n} t_{n}
$$

with $t^{n}$ being the $S O^{*}(12)$ generators. $\theta_{M}^{n}$ is called the embedding tensor in terms of which the covariant derivative implementing the minimal coupling of various fields can be written as

$$
D_{\mu}=\nabla_{\mu}-g A_{\mu}^{M} X_{M}
$$

$\nabla_{\mu}$ is the usual space-time covariant derivative including (possibly) the local $U(6)$ composite connection. The parameter $g$ is the gauge coupling constant which can be absorbed in the definition of $\theta_{M}{ }^{m}$.
In 32 representation, with $S O^{*}(12)$ generators $\left(t_{n}\right)_{M}{ }^{N}$, the embedding tensor can be described by the generalized structure constants

$$
X_{M N}^{P}=\theta_{M}{ }^{n}\left(t_{n}\right)_{N}^{P} .
$$

To define a proper gauging and preserve the full supersymmetry of the ungauged theory, the embedding tensor needs to satisfy the so-called linear and quadratic constraints given, respectively, by

$X_{(M N}{ }^{L} \Omega_{P) L}=0 \quad$ and $\quad \theta_{M}{ }^{m} \theta_{N}{ }^{n} f_{m n}{ }^{p}+X_{M N}{ }^{P} \theta_{P}{ }^{p}=0$

with $f_{m n}{ }^{p}$ being the $S O^{*}(12)$ structure constants. The former implies that the embedding tensor $\theta_{M}{ }^{m}$ is in the representation 351 of $S O^{*}(12)$, while the latter gives rise to

$$
\left[X_{M}, X_{N}\right]=-X_{M N}^{P} X_{P} .
$$

The gauge generators then form a closed subalgebra for which $X_{M N}{ }^{P}$ act as the corresponding structure constants.

As usual in gauging a supergravity theory, supersymmetry requires some modifications to the ungauged Lagrangian and supersymmetry transformations. These modifications are of first and second order in the gauge coupling constant and can be written in terms of the socalled $T$ tensor:

$$
T_{\underline{M}} \underline{N}^{\underline{P}}=\mathcal{V}_{\underline{M}}^{M} \mathcal{V}_{\underline{N}}^{N} \mathcal{V}_{P}{ }^{\underline{P}} X_{M N}{ }^{P} .
$$

The bosonic Lagrangian of the $N=6$ gauged supergravity can be written as

$$
\begin{aligned}
e^{-1} \mathcal{L}= & \frac{1}{2} R-\frac{1}{24} P_{\mu A B C D} P^{\mu A B C D} \\
& -\frac{i}{4}\left(\mathcal{N}_{\Lambda \Sigma} F_{\mu \nu}^{+\Lambda} F^{+\Sigma \mu \nu}-\overline{\mathcal{N}}_{\Lambda \Sigma} F_{\mu \nu}^{-\Lambda} F^{-\Sigma \mu \nu}\right)-V .
\end{aligned}
$$

The scalar kinetic term is given in terms of the vielbein $P_{\mu}^{A B C D}=\left(P_{\mu A B C D}\right)^{*}$ on the $S O^{*}(12) / U(6)$ coset which is defined by

$$
\begin{aligned}
P_{\mu}^{A B C D} & =\mathcal{V}^{A B M} D_{\mu} \mathcal{V}_{M}{ }^{C D} \\
& =i\left(\bar{f}^{\Lambda A B} D_{\mu} \bar{h}_{\Lambda}{ }^{C D}-\bar{h}_{\Lambda}{ }^{A B} D_{\mu} \bar{f}^{\Lambda C D}\right) .
\end{aligned}
$$

The scalar matrix appearing in the gauge kinetic terms is given by

$$
\mathcal{N}_{\Lambda \Sigma}=-\bar{h}_{\Lambda} \underline{\Lambda}\left(f^{-1}\right)_{\underline{\Lambda} \Sigma}
$$

with $\overline{\mathcal{N}}_{\Lambda \Sigma}$ being its complex conjugate. The complex self-dual and anti-self-dual gauge field strengths are defined by 


$$
F_{\mu \nu}^{ \pm \Lambda}=\frac{1}{2}\left(F_{\mu \nu}^{\Lambda} \pm \frac{i}{2} \epsilon_{\mu \nu \rho \sigma} F^{\Lambda \rho \sigma}\right)
$$

with $F_{\mu \nu}^{\Lambda}$ given by

$$
F_{\mu \nu}^{\Lambda}=\partial_{\mu} A_{\nu}^{\Lambda}-\partial_{\nu} A_{\mu}^{\Lambda}+X_{\Gamma \Sigma}^{\Lambda} A_{\mu}^{\Gamma} A_{\nu}^{\Sigma}
$$

The scalar potential is obtained from the fermion-shift matrices as follows:

$V=-2 S_{A B} S^{A B}+\frac{1}{36} N_{A}{ }^{B C D} N^{A}{ }_{B C D}+\frac{1}{6} N_{A}^{B} N_{B}^{A}$.

We note that upper and lower $S U(6)$ indices are related by complex conjugation. In terms of the various components of the $T$ tensor with the splitting of indices $\underline{\Lambda}, \underline{\Sigma}, \ldots$ as $(0,[A B])$, we have

$$
\begin{aligned}
S_{A B} & =\frac{\sqrt{2}}{5} T_{C(A, B) E}{ }^{C E}, \quad N_{A B}=-\frac{8 \sqrt{2}}{3} T_{C[A, B] E}{ }^{C E}, \\
N^{A}{ }_{B} & =-2 \sqrt{2} T_{0, B C}{ }^{A C}, \\
N_{B C D}^{A} & =-2 \sqrt{2} T_{[C D, B] E}^{A E}-\frac{1}{4} \delta_{[B}^{A} N_{C D]} .
\end{aligned}
$$

The fermionic supersymmetry transformations, with all fermionic fields vanishing, are given by

$$
\begin{gathered}
\delta \psi_{\mu A}=D_{\mu} \epsilon_{A}-S_{A B} \gamma_{\mu} \epsilon^{B}-\frac{1}{4 \sqrt{2}} \hat{F}_{\rho \sigma A B}^{+} \gamma^{\rho \sigma} \gamma_{\mu} \epsilon^{B} \\
\delta \chi_{A}=-\frac{1}{4 !} \epsilon_{A B C D E F} P_{\mu}^{B C D E} \gamma^{\mu} \epsilon^{F}+N^{B}{ }_{A} \epsilon_{B}-\frac{1}{2 \sqrt{2}} \hat{F}_{\mu \nu}^{+} \gamma^{\mu \nu} \epsilon_{A},
\end{gathered}
$$

$\delta \chi_{A B C}=-P_{\mu A B C D} \gamma^{\mu} \epsilon^{D}+N_{A B C}^{D} \epsilon_{D}-\frac{3}{2 \sqrt{2}} \hat{F}_{\mu \nu[A B}^{+} \epsilon_{C]}$.

We note here the chiralities of the fermionic fields

$$
\gamma_{5} \psi_{\mu A}=-\psi_{\mu A}, \quad \gamma_{5} \chi_{A B C}=-\chi_{A B C}, \quad \gamma_{5} \chi_{A}=-\chi_{A}
$$

with $\psi_{\mu}^{A}, \chi^{A B C}$, and $\chi^{A}$ having opposite chiralities. The tensors $\hat{F}_{\mu \nu A B}^{+}=\left(\hat{F}_{\mu \nu}^{-A B}\right)^{*}$ can be obtained from

$$
\hat{F}_{\mu \nu}^{-A B}=\mathcal{V}_{M}^{A B} G_{\mu \nu}^{-M}
$$

with

$$
G_{\mu \nu}^{M}=\left(\begin{array}{c}
F_{\mu \nu}^{\Lambda} \\
G_{\Lambda \mu \nu}
\end{array}\right)
$$

and $\quad G_{\Lambda \mu \nu}=i \epsilon_{\mu \nu \rho \sigma} \frac{\partial \mathcal{L}}{\partial F_{\rho \sigma}^{\Lambda}}$. Similarly, we have $\hat{F}_{\mu \nu}^{+}=$ $\left(\mathcal{V}_{M}{ }^{0} G_{\mu \nu}^{-M}\right)^{*}$.

The covariant derivative of $\epsilon_{A}$ is defined by

$$
D_{\mu} \epsilon_{A}=\partial_{\mu} \epsilon_{A}+\frac{1}{4} \omega_{\mu}^{a b} \gamma_{a b} \epsilon_{A}+\frac{1}{2} Q_{\mu A}{ }^{B} \epsilon_{B} .
$$

The connection $Q_{\mu A}{ }^{B}$ is given by

$Q_{\mu A}^{B}=\frac{2 i}{3}\left(h_{\Lambda A C} \partial_{\mu} \bar{f}^{\Lambda A B}-f_{A C}^{\Lambda} \partial_{\mu} \bar{h}_{\Lambda}{ }^{B C}\right)-g A_{\mu}^{M} Q_{M A}{ }^{B}$

with $Q_{M A}{ }^{B}$ obtained from

$$
Q_{M A B}^{C D}=\mathcal{V}_{A B}{ }^{P} X_{M P}{ }^{N} \mathcal{V}_{N}{ }^{C D}
$$

by the relation $Q_{M A B}{ }^{C D}=4 \delta_{[A}^{[C} Q_{M B]}^{D]}$.

In general, both electric and magnetic gauge fields can participate in the gaugings, leading to many possibilities of viable gauge groups. However, in this work, we will consider only the $S O(6)$ gauge group embedded electrically in $U(6) \subset S O^{*}(12)$. This gauging involves only electric gauge fields $A^{A B}$. In this case, we have

$$
\begin{aligned}
X_{I_{1} J_{1}, I_{2} J_{2}} I_{3} J_{3} & =4 g \delta_{\left[I_{1}\right.}^{\left[I_{3}\right.} \delta_{\left.I_{2}\right]\left[J_{2}\right.} \delta_{\left.J_{2}\right]}^{\left.J_{3}\right]} \\
\text { and } \quad X_{I_{1} J_{1}}{ }^{I_{3} J_{3}}{ }_{I_{2} J_{2}} & =-X_{I_{1} J_{1}, I_{2} J_{2}}^{I_{3} J_{3}}
\end{aligned}
$$

with all remaining components vanishing. In particular, there are no $X^{\Lambda}{ }_{M}{ }^{N}$ components which couple to magnetic gauge fields.

With the splitting of indices $\Lambda, \Sigma, \ldots=(0,[I J])$, we find from the definition (14) that

$$
\begin{aligned}
& T_{A B}{ }^{C D}=-\frac{1}{2} f^{I_{1}{ }_{0}}\left(f^{J J_{1}}{ }_{A B} \bar{h}_{I_{1} J_{1}}{ }^{C D}+h_{I_{1} J_{1}, A B} \bar{f}^{J J_{1}, C D}\right) \text {, }
\end{aligned}
$$

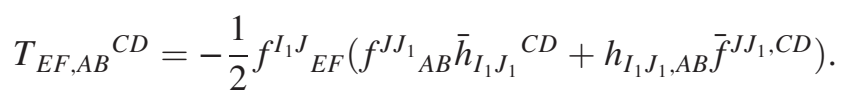

From these, it is straightforward to obtain all the fermionshift matrices and the scalar potential.

In subsequent sections, we will look for various types of supersymmetric solutions to this $N=6$ gauged supergravity with the $S O(6)$ gauge group. It has been shown in Ref. [5] that this gauged supergravity admits a supersymmetric $N=6 \mathrm{AdS}_{4}$ vacuum with the cosmological constant $V_{0}=-48 g^{2}$ when all scalars vanish. According to the AdS/CFT correspondence, this is dual to an $N=6$ SCFT in three dimensions. We will find solutions that are asymptotic to this $\mathrm{AdS}_{4}$ geometry and can be interpreted as different types of deformations of the dual $N=6 \mathrm{SCFT}$. 


\section{HOLOGRAPHIC RG FLOWS}

We first consider holographic RG flow solutions in the form of domain walls interpolating between the supersymmetric $\mathrm{AdS}_{4}$ vacuum and another $\mathrm{AdS}_{4}$ vacuum (if it exists) or a singular geometry. These solutions correspond, respectively, to RG flows of the dual UV $N=6$ SCFT to another conformal fixed point or to a nonconformal phase in the IR.

The metric ansatz is taken to be

$$
d s^{2}=e^{2 A(r)} d x_{1,2}^{2}+d r^{2}
$$

with $d x_{1,2}^{2}$ being the flat metric on three-dimensional Minkowski space. Scalar fields are allowed to depend only on the radial coordinate $r$ with all the other fields set to zero.

\section{A. Solutions with $S O(2) \times S O(4)$ symmetry}

We first consider a simple case of solutions with $S O(2) \times S O(4)$ symmetry. The embedding of $S O(6)$ implies that the scalars $\phi_{A B}$ transform as an adjoint representation of $S O(6)$. There is one singlet scalar under $S O(2) \times S O(4) \subset S O(6)$ given explicitly by

$$
\phi_{A B}=\phi\left(\delta_{A}^{1} \delta_{B}^{2}-\delta_{B}^{1} \delta_{A}^{2}\right) .
$$

We will also write

$$
\phi=\varphi e^{i \zeta}
$$

for real scalars $\varphi$ and $\zeta$ depending only on $r$.

By a straightforward computation, we find the tensor $S_{A B}$ of the form

$$
S_{A B}=2 g \cosh \varphi \delta_{A B}=\frac{1}{2} \mathcal{W} \delta_{A B} .
$$

We have introduced the "superpotential" $\mathcal{W}$ for convenience. In general, the function $\mathcal{W}$ is related to the eigenvalue of $S_{A B}$ corresponding to the unbroken supersymmetry. In the present case, $S_{A B}$ is proportional to the identity matrix, indicating that the solutions will preserve either $N=6$ supersymmetry with all $\epsilon_{A}$ nonvanishing or no supersymmetry at all. Note also that $\mathcal{W}$ has a critical point at $\varphi=0$ which is the supersymmetric $N=6$ $\mathrm{AdS}_{4}$ vacuum mentioned above.

To solve all the Bogomol'nyi-Prasad-Sommerfield (BPS) conditions, we will, as in other previous works, impose the following projector:

$$
\gamma_{\hat{r}} \epsilon_{A}=e^{i \Lambda} \epsilon^{A}
$$

for a real function $\Lambda$. Throughout the paper, we will use Majorana representation for gamma matrices in which all $\gamma^{\mu}$ are real but $\gamma_{5}$ is purely imaginary. This implies that
$\epsilon_{A}$ and $\epsilon^{A}$ are related by complex conjugation. Note also that the projector (38) relates the two chiralities of $\epsilon^{A}$, so the full flow solutions will preserve only half of the original supersymmetry or 12 supercharges.

Considering the conditions $\delta \psi_{\mu A}=0$ for $\mu=0,1,2$, we find

$$
e^{i \Lambda} A^{\prime}-\mathcal{W}=0
$$

with the prime denoting $r$ derivatives. This equation gives

$$
A^{\prime}= \pm|\mathcal{W}| \quad \text { and } \quad e^{i \Lambda}= \pm \frac{\mathcal{W}}{|\mathcal{W}|}
$$

In what follows, we will write $W=|\mathcal{W}|$ for convenience. We will also choose the upper signs in order to make the supersymmetric $\mathrm{AdS}_{4}$ critical point correspond to $r \rightarrow \infty$. Since, in this case, the superpotential is real, we simply have

$$
A^{\prime}=4 g \cosh \varphi \quad \text { and } \quad e^{i \Lambda}=1 .
$$

The condition $\delta \psi_{r A}=0$ gives the standard Killing spinors of the domain walls

$$
\epsilon_{A}=e^{A / 2} \epsilon_{A(0)}
$$

for spinors $\epsilon_{A(0)}$ satisfying Eq. (38).

Using the projection (38) in the variations $\delta \chi_{A B C}$ and $\delta \chi_{A}$ gives the following BPS equations:

$$
\varphi^{\prime}=-4 g \sinh \varphi \quad \text { and } \quad \zeta^{\prime}=0 .
$$

We have now obtained the BPS equations that solve all the supersymmetry conditions. It can also be readily verified that these equations imply the second-order field equations.

We can analytically solve the above BPS equations with the following solution:

$$
\begin{gathered}
4 g r=\ln \left(1+e^{\varphi}\right)-\ln \left(1-e^{\varphi}\right), \\
A=\varphi-\ln \left(1-e^{2 \varphi}\right) .
\end{gathered}
$$

We have neglected the integration constants in these equations, since they can be removed by shifting the radial coordinate and scaling the $x^{0,1,2}$ coordinates, respectively. As $r \rightarrow \infty$, we find that

$$
\varphi \sim e^{-4 g r} \sim e^{-r / L} \quad \text { and } \quad A \sim 4 g r \sim \frac{r}{L}
$$

with $L$ being the $\mathrm{AdS}_{4}$ radius related to the cosmological constant by 


$$
L=\sqrt{-\frac{3}{V_{0}}}=\frac{1}{4 g} .
$$

We have also taken $g>0$ for convenience.

The behavior of $\varphi$ implies that $\varphi$ is dual to a relevant operator of dimensions $\Delta=1,2$ in the dual SCFT. In addition, the solution is singular at $r \rightarrow 0$ with

$$
\varphi \sim \pm \ln (4 g r) \quad \text { and } \quad A \sim \ln (4 g r) .
$$

We then find that $\varphi \rightarrow \pm \infty$ near the singularity. From the explicit form of the scalar potential, we have

$$
V \sim-8 g^{2} e^{ \pm 2 \varphi} \rightarrow-\infty .
$$

By the criterion given in Ref. [77], we conclude that the singularity is physical. Therefore, the solution describes an RG flow from the UV $N=6$ SCFT to a nonconformal phase in the IR. The flow is driven by an operator of dimensions $\Delta=1,2$ corresponding to scalar or fermion mass terms in three dimensions. The flow breaks superconformal symmetry but preserves the full $N=6$ Poincaré supersymmetry. Moreover, the $\mathrm{R}$ symmetry $S O(6)$ is broken to $S O(2) \times S O(4)$ subgroup. This is precisely in agreement with the field theory result given in Ref. [25]. We then expect the solution to describe mass deformations of the three-dimensional $N=6$ SCFT.

\section{B. Solutions with $U(3)$ symmetry}

We now consider another residual symmetry, namely, $U(3) \sim S U(3) \times U(1) \subset S O(6)$. The $U(3)$ generators in the fundamental representation of $S O(6)$ can be written as

$$
X=\left(\begin{array}{cc}
A_{3 \times 3} & S_{3 \times 3} \\
-S_{3 \times 3} & A_{3 \times 3}
\end{array}\right)
$$

in which $A_{3 \times 3}$ and $S_{3 \times 3}$ are antisymmetric and symmetric $3 \times 3$ matrices, respectively. The matrices $A_{3 \times 3}$ generate an $S O(3) \subset S U(3)$ which is a diagonal subgroup of $S O(3) \times S O(3) \subset S O(6)$. The $U(1)$ factor corresponds to $S_{3 \times 3}=\mathbb{I}_{3}$. There is only one $U(3)$ singlet scalar given by

$$
\phi_{A B}=\left(\begin{array}{cc}
0_{3 \times 3} & \phi \mathbb{I}_{3} \\
-\phi \mathbb{I}_{3} & 0_{3 \times 3}
\end{array}\right)=\phi J_{A B} .
$$

The matrix $J_{A B}$ is identified with the Kähler form of $C P^{3}$ on which the ten-dimensional type IIA theory compactifies [5].

By writing $\phi=\varphi e^{i \zeta}$ and repeating the same analysis as in the previous case, we find the scalar potential

$$
V=-24 g^{2} e^{-2 \varphi}\left(1+e^{4 \varphi}\right),
$$

which is exactly the same as that given in Ref. [5]. As in the $S O(2) \times S O(4)$ case, this potential admits an $N=6 \mathrm{AdS}_{4}$ critical point at $\varphi=0$ dual to an $N=6$ SCFT in three dimensions.

The matrix $S_{A B}$ is proportional to the identity

$$
S_{A B}=\frac{1}{2} \mathcal{W} \delta_{A B}
$$

with a complex superpotential

$\mathcal{W}=\frac{1}{2} e^{-3 \varphi-i \zeta}\left[\left(e^{6 \varphi}+3 e^{2 \varphi}\right)\left(1+e^{i \zeta}\right)+\left(1+e^{4 \varphi}\right)\left(e^{i \zeta}-1\right)\right]$

The variations $\delta \chi_{A}$ and $\delta \chi_{A B C}$ lead to

$$
\begin{aligned}
& e^{-i \Lambda}\left(2 \varphi^{\prime} \pm i \sinh (2 \varphi) \zeta^{\prime}\right) \\
& \quad=-g e^{-3 \varphi}\left(e^{4 \varphi}-1\right)\left[1-e^{i \zeta}+e^{2 \varphi}\left(1+e^{i \zeta}\right)\right],
\end{aligned}
$$

which implies $\zeta^{\prime}=0$. It turns out that $\zeta^{\prime}=0$ is also required by the field equations. For constant $\zeta=\zeta_{0}$, we have verified that all the resulting BPS equations are compactible with the field equations. In the following analysis, we will set $\zeta_{0}=0$ and end up with the BPS equations

$\varphi^{\prime}=-e^{-\varphi}\left(e^{4 \varphi}-1\right) \quad$ and $\quad A^{\prime}=g e^{-\varphi}\left(3+e^{4 \varphi}\right)$.

The solution can be readily obtained:

$$
\begin{gathered}
A=3 \varphi-\ln \left(1-e^{4 \varphi}\right), \\
4 g r=2 \tan ^{-1} e^{\varphi}-\ln \left(1-e^{\varphi}\right)+\ln \left(1+e^{\varphi}\right) .
\end{gathered}
$$

As in the previous case, the solution is asymptotic to the supersymmetric $\mathrm{AdS}_{4}$ with $\varphi$ dual to an operator of dimensions $\Delta=1,2$, while at $r=0$ the solution is singular with

$$
\varphi \sim \ln (g r) \quad \text { and } \quad A \sim 3 \varphi \sim 3 \ln (g r)
$$

and

$$
\varphi \sim-\ln (g r) \quad \text { and } \quad A \sim-\varphi \sim \ln (g r) .
$$

Both of these give

$$
V \sim-24 g^{2} e^{ \pm 2 \varphi} \rightarrow-\infty,
$$

so the two singularities are physical. We can accordingly interpret the solution as a holographic dual of RG flows from the $N=6$ SCFT to nonconformal phases in the IR. The flow preserves $N=6$ Poincaré supersymmetry in three dimensions as in the $S O(2) \times S O(4)$ case but breaks the $S O(6) \mathrm{R}$ symmetry to $U(3)$. It would be interesting to 
identify the corresponding mass deformations in the dual $N=6$ SCFT similar to the $S O(2) \times S O(4)$ case.

\section{Solutions with $S O(2) \times S O(2) \times S O(2)$ symmetry}

To obtain more interesting and more complicated solutions, we consider solutions with a smaller symmetry, namely, $S O(2) \times S O(2) \times S O(2) \subset S O(6)$ symmetry. There are three complex scalars which are singlets under this $S O(2) \times S O(2) \times S O(2)$. The explicit parametrization of these singlets can be written as

$$
\phi_{A B}=\left(\begin{array}{ccc}
\phi_{1} i \sigma_{2} & 0_{2 \times 2} & 0_{2 \times 2} \\
0_{2 \times 2} & \phi_{2} i \sigma_{2} & 0_{2 \times 2} \\
0_{2 \times 2} & 0_{2 \times 2} & \phi_{3} i \sigma_{2}
\end{array}\right) .
$$

By setting

$$
\phi_{\alpha}=\varphi_{\alpha} e^{i \zeta_{\alpha}}, \quad \alpha=1,2,3,
$$

we find the scalar potential

$V=-16 g^{2}\left[\cosh \left(2 \varphi_{1}\right)+\cosh \left(2 \varphi_{2}\right)+\cosh \left(2 \varphi_{3}\right)\right]$.

It is clearly seen that this potential admits only one critical point at $\varphi_{1}=\varphi_{2}=\varphi_{3}=0$ which is the aforementioned $N=6 \mathrm{AdS}_{4}$ vacuum.

The matrix $S_{A B}$ is given by

$$
S_{A B}=\frac{1}{2}\left(\begin{array}{ccc}
\mathcal{W}_{1} \mathbb{I}_{2} & 0_{2 \times 2} & 0_{2 \times 2} \\
0_{2 \times 2} & \mathcal{W}_{2} \mathbb{I}_{2} & 0_{2 \times 2} \\
0_{2 \times 2} & 0_{2 \times 2} & \mathcal{W}_{3} \mathbb{I}_{2}
\end{array}\right)
$$

with

$$
\begin{aligned}
\mathcal{W}_{1}= & \frac{1}{2} g e^{-\varphi_{1}-\varphi_{2}-\varphi_{3}} \\
& \times\left[e^{i\left(\zeta_{1}-\zeta_{2}-\zeta_{3}\right)}\left(e^{2 \varphi_{1}}-1\right)\left(e^{2 \varphi_{2}}-1\right)\left(e^{2 \varphi_{3}}-1\right)\right. \\
& \left.-\left(1+e^{2 \varphi_{1}}\right)\left(1+e^{2 \varphi_{2}}\right)\left(1+e^{2 \varphi_{3}}\right)\right] .
\end{aligned}
$$

$\mathcal{W}_{2}$ and $\mathcal{W}_{3}$ take a similar form with the phase $e^{i\left(\zeta_{1}-\zeta_{2}-\zeta_{3}\right)}$ replaced by $e^{i\left(\zeta_{2}-\zeta_{1}-\zeta_{3}\right)}$ and $e^{i\left(\zeta_{3}-\zeta_{1}-\zeta_{2}\right)}$, respectively.

It turns out that none of these $\mathcal{W}_{\alpha}$ gives rise to the superpotential in term of which the scalar potential (64) can be written unless $\zeta_{1}=\zeta_{2}=\zeta_{3}=0$. This is also implied by the consistency between the resulting BPS equations and the field equations. We now set $\zeta_{1}=\zeta_{2}=\zeta_{3}=0$, resulting in $e^{i \Lambda}= \pm 1$, and obtain the following BPS equations:

$\varphi_{1}^{\prime}=-g e^{-\varphi_{1}-\varphi_{2}-\varphi_{3}}\left[e^{2\left(\varphi_{1}+\varphi_{2}\right)}+e^{2\left(\varphi_{1}+\varphi_{3}\right)}-e^{2\left(\varphi_{2}+\varphi_{3}\right)}-1\right]$,

$\varphi_{2}^{\prime}=-g e^{-\varphi_{1}-\varphi_{2}-\varphi_{3}}\left[e^{2\left(\varphi_{1}+\varphi_{2}\right)}+e^{2\left(\varphi_{2}+\varphi_{3}\right)}-e^{2\left(\varphi_{1}+\varphi_{3}\right)}-1\right]$, $\varphi_{3}^{\prime}=-g e^{-\varphi_{1}-\varphi_{2}-\varphi_{3}}\left[e^{2\left(\varphi_{1}+\varphi_{3}\right)}+e^{2\left(\varphi_{2}+\varphi_{3}\right)}-e^{2\left(\varphi_{1}+\varphi_{2}\right)}-1\right]$,

$A^{\prime}=g e^{-\varphi_{1}-\varphi_{2}-\varphi_{3}}\left[e^{2\left(\varphi_{1}+\varphi_{2}\right)}+e^{2\left(\varphi_{1}+\varphi_{3}\right)}+e^{2\left(\varphi_{3}+\varphi_{3}\right)}+1\right]$.

To find the solution to these equations, we first take a linear combination:

$$
\varphi_{1}^{\prime}+\varphi_{2}^{\prime}=-2 g e^{-\varphi_{1}-\varphi_{2}-\varphi_{3}}\left(e^{2\left(\varphi_{1}+\varphi_{2}\right)}-1\right) .
$$

After changing to a new radial coordinate $\rho$ defined by

$$
\frac{d \rho}{d r}=e^{-\varphi_{1}-\varphi_{2}-\varphi_{3}},
$$

we find

$$
\varphi_{2}=2 g \rho-\varphi_{1}-\frac{1}{2} \ln \left(e^{4 g \rho}+C_{2}\right)
$$

for a constant $C_{2}$.

Similarly, taking a linear combination $\varphi_{1}^{\prime}+\varphi_{3}^{\prime}$ gives rise to

$$
\varphi_{3}=2 g \rho-\varphi_{1}-\frac{1}{2} \ln \left(e^{4 g \rho}+C_{3}\right) .
$$

Using these results in Eq. (67), we find

$$
\varphi_{1}=\frac{1}{4} \ln \left[\frac{e^{4 g \rho}\left(e^{4 g \rho}+C_{1}\right)}{\left(e^{4 g \rho}+C_{2}\right)\left(e^{4 g \rho}+C_{3}\right)}\right] .
$$

Finally, with all these results, the solution for $A$ is given by

$$
\begin{aligned}
A= & g \rho+\frac{1}{4} \ln \left(e^{4 g \rho}+C_{1}\right)+\frac{1}{4} \ln \left(e^{4 g \rho}+C_{2}\right) \\
& +\frac{1}{4} \ln \left(e^{4 g \rho}+C_{3}\right) .
\end{aligned}
$$

We now look at the behavior of the solution as $\varphi_{\alpha} \sim 0$ which gives $\rho \sim r$ and

$\varphi_{1} \sim \frac{1}{4}\left(C_{1}-C_{2}-C_{3}\right) e^{-4 g \rho}, \quad \varphi_{2,3} \sim-\frac{1}{4}\left(C_{1}-C_{3,2}\right) e^{-4 g \rho}$,

$$
A \sim 4 g \rho \text {. }
$$

This is the expected behavior of the solution asymptotic to the supersymmetric $\mathrm{AdS}_{4}$ vacuum. As in the previous cases, the solution is singular as $4 g \rho \rightarrow \ln \left(-C_{\alpha}\right)$. For $C_{1} \neq C_{2} \neq C_{3}$, there are three possibilities. 
(i) $C_{1}<C_{2,3}$. - In this case, the solution is singular when $4 g \rho \rightarrow \ln \left(-C_{1}\right)$ with

$$
\begin{aligned}
\varphi_{1} & \sim \frac{1}{4} \ln \left(4 g \rho-\tilde{C}_{1}\right), \quad \tilde{C}_{1}=\ln \left(-C_{1}\right), \\
\varphi_{2,3} & \sim-\varphi_{1}, \quad A \sim \varphi_{1} .
\end{aligned}
$$

(ii) $C_{2}<C_{1,3}$ or $C_{3}<C_{1,2}$. - In this case, we find

$$
\begin{aligned}
\varphi_{1} & \sim-\frac{1}{4} \ln \left(4 g \rho-\tilde{C}_{2,3}\right), \quad \tilde{C}_{2,3}=\ln \left(-C_{2,3}\right), \\
\varphi_{2,3} & \sim \varphi_{1}, \quad A \sim-\varphi_{1} .
\end{aligned}
$$

In the first case, we have $\varphi_{1} \rightarrow-\infty$ and $\varphi_{2,3} \rightarrow \infty$, while in the second case, the solution gives $\varphi_{1,2,3} \rightarrow \infty$. It can be easily verified that all of these behaviors lead to $V \rightarrow-\infty$, so the singularities are physically acceptable. The solution then describes different types of mass deformations within the dual $N=6$ SCFT to nonconformal phases with $S O(2) \times S O(2) \times S O(2)$ symmetry. The solution also preserves $N=6$ Poincaré supersymmetry as in the previous cases.

\section{Solutions with $S O(3)$ symmetry}

We further reduce the residual symmetry to $S O(3) \subset$ $S O(3) \times S O(3) \subset S O(6)$ generated by the antisymmetric matrices $A_{3 \times 3}$ in the upper-left block of Eq. (50). There are three singlet scalars parametrized by

$$
\phi_{A B}=\left(\begin{array}{ll}
0_{3 \times 3} & 0_{3 \times 3} \\
0_{3 \times 3} & \hat{A}_{3 \times 3}
\end{array}\right)
$$

with

$$
\hat{A}=\left(\begin{array}{ccc}
0 & \tilde{\phi}_{1} & \tilde{\phi}_{2} \\
-\tilde{\phi}_{1} & 0 & \tilde{\phi}_{3} \\
-\tilde{\phi}_{2} & -\tilde{\phi}_{3} & 0
\end{array}\right)
$$

By writing $\tilde{\phi}_{\alpha}=\varphi_{\alpha} e^{i \zeta_{\alpha}}$ with

$\varphi_{1}=\Phi \cos \theta, \quad \varphi_{2}=\Phi \sin \theta \cos \vartheta, \quad \varphi_{3}=\Phi \sin \theta \sin \vartheta$

and

$$
\zeta_{1}=\zeta, \quad \zeta_{2}=\zeta+\eta, \quad \zeta_{3}=\zeta+\xi
$$

we find the scalar potential

$$
\begin{aligned}
V= & -g^{2}\left[16 \cos ^{4} \theta(2+\cosh 2 \Phi)+16 \cosh ^{4} \theta \sin ^{4} \theta(2+\cosh 2 \Phi)+16 \sin ^{4} \theta \sin ^{4} \vartheta(2+\cosh 2 \Phi)-\cos ^{2} \theta \sin ^{2} \theta \cos ^{2} \vartheta\right. \\
& \times\left(\cosh 4 \Phi-8 \cos 2 \eta \sinh ^{4} \Phi-36 \cosh 2 \Phi-61\right)+\sin ^{2} \theta \sin ^{2} \vartheta \times\left[8 \sinh ^{4} \Phi\left(\cos ^{2} \theta \cos 2 \xi+\cos ^{2} \vartheta \sin ^{2} \theta \cos [2(\eta-\xi)]\right)\right. \\
& \left.\left.+(61+36 \cosh 2 \Phi-\cosh 4 \Phi)\left(\cos ^{2} \theta+\cos ^{2} \vartheta \sin ^{2} \theta\right)\right]\right] .
\end{aligned}
$$

In this case, the scalar potential depends on phases of the complex scalars, and the analysis is more complicated. To make the analysis more traceable, we will further truncate to two scalars by setting $\vartheta=0$ and $\xi=-\zeta$. This is equivalent to setting $\tilde{\phi}_{3}=0$. We now begin with the eigenvalues of the matrix $S_{A B}$. These are of the form, after diagonalization,

$$
S_{A B}^{\mathrm{diag}}=\operatorname{diag}\left(-2 g \cosh \Phi_{\times 4}, \frac{1}{2} \mathcal{W}_{+}, \frac{1}{2} \mathcal{W}_{-}\right)
$$

with $\mathcal{W}_{ \pm}$given by

$$
\mathcal{W}_{ \pm}=2 g(\cos 2 \eta+2 \sin \eta) \sinh ^{4} \frac{\Phi}{2}(\cos 4 \theta \sin \eta \pm i \sin 2 \theta)-\frac{1}{4} g(3+12 \cosh \Phi+\cosh 2 \Phi) .
$$

The corresponding eigenvectors are

$$
\hat{\epsilon}_{ \pm}=-\frac{1}{2} \sec 2 \theta\left(2 \cos \eta \sin 2 \theta \mp \sqrt{3+\cos 2 \eta+2 \cos 4 \theta \sin ^{2} \eta}\right) \epsilon_{5}+\epsilon_{6} .
$$

The scalar kinetic term is given by

$$
\begin{aligned}
\mathcal{L}_{\text {kin }} & =-\frac{1}{2} G_{\alpha \beta} \phi^{\alpha \prime} \phi^{\beta \prime} \\
& =-\Phi^{\prime 2}-\sinh ^{2} \Phi \theta^{\prime 2}-\frac{1}{4} \sinh ^{2} 2 \Phi \zeta^{\prime 2}-\frac{1}{2} \sin ^{2} \theta \sinh ^{2} 2 \Phi \zeta^{\prime} \eta^{\prime}-\frac{1}{4} \sin ^{2} \theta \sinh ^{2} \Phi\left(3+\cosh 2 \Phi-2 \cos 2 \theta \sinh ^{2} \Phi\right) \eta^{\prime 2}
\end{aligned}
$$


with $\phi^{\alpha}=(\Phi, \theta, \zeta, \eta)$. It is useful to give an explicit form of the inverse of $G_{\alpha \beta}$ here:

$$
G^{\alpha \beta}=-\frac{1}{2}\left(\begin{array}{cccc}
1 & 0 & 0 & 0 \\
0 & \operatorname{csch}^{2} \Phi & 0 & 0 \\
0 & 0 & -\operatorname{sech}^{2} \Phi+\operatorname{csch}^{2} \Phi \sec ^{2} \theta & -\operatorname{csch}^{2} \Phi \sec ^{2} \theta \\
0 & 0 & -\operatorname{csch}^{2} \Phi \sec ^{2} \theta & 4 \csc ^{2} 2 \theta \operatorname{csch} \Phi
\end{array}\right) .
$$

The scalar potential can be written in term of the real superpotential $W=\left|\mathcal{W}_{+}\right|=\left|\mathcal{W}_{-}\right|$as

$$
\begin{aligned}
V & =-2 G^{\alpha \beta} \frac{\partial W}{\partial \phi^{\alpha}} \frac{\partial W}{\partial \phi^{\beta}}-3 W^{2} \\
& =g^{2}\left[\cos ^{2} \theta \sin ^{2} \theta\left(\cosh 4 \Phi-8 \cos 2 \eta \sinh ^{4} \Phi-36 \cosh 2 \Phi-61\right)-4(3+\cos 4 \theta)(2+\cosh 2 \Phi)\right] .
\end{aligned}
$$

After setting $\epsilon^{1,2,3,4}=0$ and imposing the projection conditions

$$
\gamma_{\hat{r}} \epsilon_{ \pm}=e^{ \pm i \Lambda} \epsilon^{ \pm} \quad \text { with } \quad e^{ \pm i \Lambda}=\frac{\mathcal{W}_{ \pm}}{W}
$$

we find the following BPS equations:

$$
\begin{gathered}
\Phi^{\prime}=\frac{1}{16 W} g^{2}\left[8 \sinh ^{3} \Phi \cosh \Phi\left(\cos 2 \eta+2 \cos 4 \theta \sin ^{3} \eta\right)-30 \sin 2 \Phi-\sinh 4 \Phi\right] \\
\theta^{\prime}=-\frac{1}{W} g^{2} \sin ^{2} \eta \sin 4 \theta \sinh ^{2} \Phi
\end{gathered}
$$

$$
\begin{gathered}
\zeta^{\prime}=\frac{2}{W} g^{2} \sin 2 \eta \sin ^{2} \theta \sinh ^{2} \Phi, \\
\eta^{\prime}=-\frac{2}{W} g^{2} \sin 2 \eta \sinh ^{2} \Phi, \\
A^{\prime}=W .
\end{gathered}
$$

The flow equations for the scalars can be written in a compact form as

$$
\phi^{\alpha \prime}=2 G^{\alpha \beta} \frac{\partial W}{\partial \phi^{\beta}}
$$

It can straightforwardly be verified that all these equations satisfy the second-order field equations. We also see that, from these equations, there is only one supersymmetric critical point, with $\Phi^{\prime}=\theta^{\prime}=\zeta^{\prime}=\eta^{\prime}=0$, at $\phi^{\alpha}=0$.

We note that, although the superpotential and the scalar potential do not depend on $\zeta, \zeta^{\prime}$ is still nonvanishing due to the mixed terms between $\zeta$ and $\eta$ in the matrix $G_{\alpha \beta}$. Moreover, further truncations such as $\eta=0$ or $\theta=0$ will lead to the BPS equations in the case of $N=6$ supersymmetry with all six eigenvalues of $S_{A B}$ leading to

$$
\mathcal{W}=4 g \cosh \Phi .
$$

This is very similar to the $N=5$ gauged supergravity studied in Ref. [51] in which the differences in the phases of the scalars are crucial for breaking the original supersymmetry to a lower amount.

We now look at the solution to the above equations. Combining $\eta^{\prime}$ and $\theta^{\prime}$ equations gives

$$
\frac{d \theta}{d \eta}=\frac{1}{4} \sin 4 \theta \tan \eta
$$

with the solution given by

$$
\cot 2 \theta=C_{1} \cos \eta
$$

Similarly, taking the combination between $\zeta^{\prime}$ and $\eta^{\prime}$ equations leads to

$$
\frac{d \zeta}{d \eta}=-\sin ^{2} \theta
$$

After using the above solution for $\theta$, we find the solution

$\zeta=\zeta_{0}-\frac{\eta}{2}+\frac{\sqrt{C_{1}^{2}+\sec ^{2} \eta} \cos \eta \tan ^{1} \frac{\sqrt{2} C_{1} \sin \eta}{\sqrt{2+C_{1}^{2}(1+\cos 2 \eta)}}}{\sqrt{4+2 C_{1}^{2}(1+\cos 2 \eta)}}$

for constant $\zeta_{0}$. 
Combining $\Phi^{\prime}$ and $\eta^{\prime}$ equations by taking into account all the previous results together with a redefinition

$$
\tilde{\Phi}=\sinh \Phi
$$

we find

$$
\frac{d \tilde{\Phi}}{d \eta}=\csc 2 \eta\left(1+\tilde{\Phi}^{2}\right)\left(\frac{\tilde{\Phi} \tan ^{2} \eta}{C_{1}^{2}+\sec ^{2} \eta}+\frac{2}{\tilde{\Phi}}\right)
$$

whose solution is given by

$$
\frac{\tilde{\Phi}^{2}}{4}=-\frac{1+C_{1}^{2} \cos ^{2} \eta-C_{2} \sqrt{(1+\cos 2 \eta)\left(2+C_{1}^{2}(1+\cos 2 \eta)\right)}}{3+4 C_{1}^{2} \cos ^{2} \eta+\cos 2 \eta-4 C_{2} \sqrt{(1+\cos 2 \eta)\left(2+C_{1}^{2}(1+\cos 2 \eta)\right)}} .
$$

Using all these results in the $\eta^{\prime}$ equation, we find the solution for $\eta(r)$ implicitly from

$$
8 g r=\sinh ^{-1}\left[2 C_{2} \sqrt{\frac{\Xi-1}{\left(1+C_{1}^{2}\right)\left[2+\left(C_{1}^{2}-4 C_{2}^{2}\right)(1+\Xi)\right]}}\right]-\tanh ^{-1} \sqrt{\frac{\left(1+C_{1}^{2}-4 C_{2}^{2}\right)(1+\Xi)}{\Xi-1}},
$$

in which we have defined

$$
\Xi=\cos 2 \eta
$$

Finally, we can find the solution for $A(\Xi)$ as

$$
\begin{aligned}
A= & \frac{1}{4}\left(\tanh ^{-1} \alpha_{+}-\tanh ^{-1} \alpha_{-}\right)-\frac{1}{2} \tanh ^{-1}\left[2 C_{2} \sqrt{\frac{\Xi+1}{2+C_{1}^{2}(1+\Xi)}}\right] \\
& -\frac{1}{8} \ln \left[4 C_{1}^{4}(1+\Xi)^{2}+(3+\Xi)^{2}-4(1+\Xi)\left[8 C_{2}^{2}+C_{1}^{2}\left(4 C_{2}^{2}(1+\Xi)-3-\Xi\right)\right]\right]+\frac{1}{4} \ln \left[2+(1+\Xi)\left(C_{1}^{2}-4 C_{2}^{2}\right)\right]
\end{aligned}
$$

with $\alpha_{ \pm}$defined by

$$
\alpha_{ \pm}=\sqrt{-\frac{2+C_{1}^{2}(1+\Xi)}{(1+\Xi)\left[1+C_{1}^{2}-4 C_{2}\left(2 C_{2}+ \pm \sqrt{4 C_{2}^{2}-C_{1}^{2}-1}\right)\right.}}
$$

The solution preserves $N=2$ supersymmetry and breaks the $S O(6)$ R symmetry to $S O(3)$. The solution is singular when

$$
\cos ^{2} \eta=-\frac{1}{1+2 C_{1}^{2}-8 C_{2}^{2} \pm 4 C_{2} \sqrt{4 C_{2}^{2}-C_{1}^{2}-1}}
$$

This gives $\tilde{\Phi} \rightarrow \pm \infty$ or $\Phi \rightarrow \pm \infty$, which, in turn, leads to

$$
V \rightarrow g^{2} e^{4|\Phi|} \cos ^{2} \theta \sin ^{2} \eta \sin ^{2} \theta
$$

We can see that the scalar potential is unbounded from above, $V \rightarrow+\infty$, unless $\theta=0$ or $\eta=0$, both of which give the $N=6$ solution as previously mentioned. Therefore, the
IR singularities of the $N=2$ solutions are unphysical by the criterion of Ref. [77].

\section{SUPERSYMMETRIC JANUS SOLUTIONS}

In this section, we consider supersymmetric Janus solutions in the form of curved domain walls. The solutions can be obtained from an $\mathrm{AdS}_{3}$-sliced domain wall ansatz

$$
d s^{2}=e^{2 A}\left(e^{2 \xi / \ell} d x_{1,1}^{2}+d \xi^{2}\right)+d r^{2} .
$$

Since the analysis closely follows that given in Ref. [52] (see also [53]), we will not repeat all the detail here but mainly review relevant results for deriving the corresponding BPS equations. 
Compared to the RG flow case, the BPS equations will be modified by the curvature of the three-dimensional slices. In addition, as pointed out in Ref. [52], the existence of Janus solutions requires nonvanishing pseudoscalars, resulting in a more complicated set of BPS equations in contrast to the simple flat domain wall or RG flow case. We begin with the conditions $\delta \psi_{\hat{\mu}}^{i}=0$ for $\hat{\mu}=0,1$, which give

$$
A^{\prime} \gamma_{\hat{r}} \epsilon_{A}+\frac{1}{\ell} e^{-A} \gamma_{\hat{\xi}} \epsilon_{A}-\mathcal{W} \epsilon^{A}=0 .
$$

This leads to the following BPS equation:

$$
A^{\prime 2}=W^{2}-\frac{1}{\ell^{2}} e^{-2 A}
$$

for $W=|\mathcal{W}|$. We still use the $\gamma_{\hat{r}}$ projection given in Eq. (38). Imposing the $\gamma_{\hat{\xi}}$ projection of the form

$$
\gamma_{\hat{\xi}} \epsilon_{A}=i \kappa e^{i \Lambda} \epsilon^{A}
$$

with $\kappa^{2}=1$, we can solve the condition (113) for the $\gamma_{\hat{r}}$ projector, leading to the phase factor

$$
e^{i \Lambda}=\frac{A^{\prime}}{W}+\frac{i \kappa}{\ell} \frac{e^{-A}}{W}
$$

for real $\mathcal{W}$ and

$$
e^{i \Lambda}=\frac{\mathcal{W}}{A^{\prime}+\frac{i \kappa}{\ell} e^{-A}}
$$

for complex $\mathcal{W}$. We also note that the constant $\kappa= \pm 1$ corresponds to the chiralities of the Killing spinors on the two-dimensional conformal defects described by the $\mathrm{AdS}_{3}$ slices. Finally, the conditions $\delta \psi_{\tilde{\xi}}^{A}=0$ and $\delta \psi_{\hat{r}}^{A}=0$ can be solved to obtain the explicit form of the Killing spinors (see details in [52]):

$$
\epsilon_{A}=e^{(A / 2)+(\xi / 2 \ell)+i(\Lambda / 2)} \varepsilon_{A}^{(0)},
$$

in which the constant spinors $\varepsilon_{A}^{(0)}$ could possibly have an $r$-dependent phase and satisfy

$$
\gamma_{\hat{r}} \varepsilon_{A}^{(0)}=\varepsilon^{(0) A} \quad \text { and } \quad \gamma_{\hat{\xi}} \varepsilon_{A}^{(0)}=i \kappa \varepsilon^{(0) A} .
$$

It turns out that among the previously considered cases only $S O(2) \times S O(4)$ and $S O(3)$ symmetric scalars can possibly possess supersymmetric Janus solutions. This is mainly a consequence of the consistency in turning on nonvanishing pseudoscalars. For the $S O(3)$ case, the analysis is highly complicated as already seen in the case of RG flows considered in the previous section. Therefore, we will give only the Janus solution with $S O(2) \times S O(4)$ symmetry. This case is more traceable, and it turns out that the solution can be analytically obtained.

\section{A. Janus solutions with $S O(2) \times S O(4)$ symmetry}

In this case, the superpotential is real, so we will use the phase $e^{i \Lambda}$ from Eq. (116). In general, since $\epsilon^{1,2}$ and $\epsilon^{A}$ with $A=3,4,5,6$ transform differently under $S O(2) \times S O(4)$, namely, as $(\mathbf{2}, \mathbf{1})+(\mathbf{1}, \mathbf{4})$, the two sets of Killing spinors can satisfy different projectors. We find that, in order to obtain a consistent set of BPS equations, we need to choose opposite signs of $\kappa$ for $\epsilon^{1,2}$ and $\epsilon^{3, \ldots, 6}$. Therefore, the surface defect will preserve $N=(2,4)$ or $N=(4,2)$ superconformal symmetry.

With the superpotential

$$
\mathcal{W}=4 g \cosh \varphi,
$$

we find the following BPS equations:

$$
\begin{gathered}
\varphi^{\prime}=-\frac{8 g^{2} \ell^{2} A^{\prime} e^{2 A} \sinh (2 \varphi)}{1+\ell^{2} A^{\prime 2} e^{2 A}}, \\
\zeta^{\prime}=-\frac{16 g^{2} \kappa \ell e^{A}}{1+\ell^{2} A^{\prime 2} e^{2 A}}, \\
A^{\prime 2}+\frac{e^{-2 A}}{\ell^{2}}=16 g^{2} \ell^{2} \cosh ^{2} \varphi .
\end{gathered}
$$

It should be noted that, for $\ell \rightarrow \infty$, these equations reduce to those of the RG flow studied in the previous section. Furthermore, these equations take a very similar form to the $S O(4)$ symmetric Janus solution in $N=5$ gauged supergravity studied in Ref. [51].

By taking $\varphi$ as an independent variable, we can solve for $A(\varphi)$ and $\zeta(\varphi)$. The complete solution is given by

$$
\begin{gathered}
A=C-\ln \sinh \varphi \\
\cosh (2 \varphi)=\frac{32 g^{2} \ell^{2} \tanh ^{2}\left[4 g\left(r-r_{0}\right)\right]}{16 g^{2} \ell^{2}-1}, \\
\kappa \tan \zeta=-\sqrt{1-16 g^{2} \ell^{2}} \sinh \left[4 g\left(r-r_{0}\right)\right]
\end{gathered}
$$

for constants $C$ and $r_{0}$. This solution takes the same form as the solution given in Refs. [52,51,53], respectively, in $N=8,5$, and 3 gauged supergravities. We also note the unbroken supersymmetries on the conformal defects in these cases as follows: $N=(4,4), N=(4,1)$, and $N=(2,1)$. All of these solutions should be related by truncations of $N=8$ gauged supergravity to $N=3$ and $N=5,6$ theories. This indicates that the $N=(4,4)$ Janus solution of Ref. [52] survives in the truncation to $N=3,5$, 6 gauged supergravities. According to the AdS/CFT correspondence, we then expect the dual $N=3,5,6$ 
SCFTs to possess the same two-dimensional conformal defect as in the $N=8$ SCFT.

We end this section by giving a brief comment on the possible $S O(3)$ symmetric Janus solution. A partial analysis shows that there appears to be no obstruction in obtaining the BPS equations for Janus solutions in this case as in a similar analysis of $N=5$ theory in Ref. [51]. Therefore, we expect a supersymmetric $N=2$ Janus solution with $S O(3)$ symmetry to exist in $N=6$ gauged supergravity as well. Since the analysis is far more complicated than the $S O(2) \times S O(4)$ case, we refrain from giving any definite result here.

\section{SUPERSYMMETRIC AdS 4 BLACK HOLES}

In this section, we look for supersymmetric $\mathrm{AdS}_{4}$ black holes in the form of curved-domain wall solutions interpolating between a locally asymptotically $\mathrm{AdS}_{4}$ and $\mathrm{AdS}_{2} \times \Sigma^{2}$ geometries. The $\mathrm{AdS}_{2} \times \Sigma^{2}$ space describes the near-horizon geometry of the black holes. In the following analysis, we will consider only the cases of $\Sigma^{2}$ being a twosphere $\left(S^{2}\right)$ and a hyperbolic space $\left(H^{2}\right)$.

The metric ansatz is taken to be

$$
d s^{2}=-e^{2 f(r)} d t^{2}+d r^{2}+e^{2 h(r)}\left(d \theta^{2}+F^{2}(\theta) d \phi^{2}\right)
$$

with the function $F(\theta)$ defined by

$$
F(\theta)= \begin{cases}\sin \theta, & \Sigma_{2}=S^{2}, \\ \sinh \theta, & \Sigma_{2}=H^{2} .\end{cases}
$$

It is straightforward to derive nonvanishing components of the spin connection

$$
\begin{array}{rlrl}
\omega^{\hat{\hat{t}}} & =f^{\prime} e^{\hat{t}}, & \omega^{\hat{\theta} \hat{r}}=h^{\prime} e^{\hat{\theta}}, \\
\omega^{\hat{\phi} \hat{r}}=h^{\prime} e^{\hat{\phi}}, & \omega^{\hat{\theta} \hat{\phi}}=\frac{F^{\prime}}{F} e^{-h} e^{\hat{\phi}}
\end{array}
$$

with $F^{\prime}(\theta)=\frac{d F}{d \theta}$.

In general, the curvature of the $\Sigma^{2}$ part on the world volume of the domain wall will completely break supersymmetry. However, it is well known that some amount of supersymmetry can be preserved by performing a topological twist. This can be achieved by turning on some gauge fields along $\Sigma^{2}$ in such a way that the corresponding spin connection, $\omega^{\hat{\theta} \hat{\phi}}$ in the above metric, is canceled. This turns the covariant derivative of $\epsilon_{A}$ along $\Sigma^{2}$ into a partial one. The resulting Killing spinors are accordingly given by spinors that are independent of the $\Sigma^{2}$ coordinates.

\section{A. $S O(2) \times S O(2) \times S O(2)$ symmetric solutions}

We first consider the $S O(2) \times S O(2) \times S O(2)$ twist with the following ansatz for the gauge fields:

$$
\begin{aligned}
& A^{12}=A_{1}(r) d t-p_{1} F^{\prime}(\theta) d \phi, \\
& A^{34}=A_{2}(r) d t-p_{2} F^{\prime}(\theta) d \phi, \\
& A^{56}=A_{3}(r) d t-p_{3} F^{\prime}(\theta) d \phi .
\end{aligned}
$$

The constants $p_{i}, i=1,2,3$, are identified with magnetic charges. The corresponding field strengths are given by

$$
\begin{aligned}
& F^{12}=A_{1}^{\prime} d r \wedge d t+\kappa p_{1} F(\theta) d \theta \wedge d \phi, \\
& F^{34}=A_{2}^{\prime} d r \wedge d t+\kappa p_{2} F(\theta) d \theta \wedge d \phi, \\
& F^{56}=A_{3}^{\prime} d r \wedge d t+\kappa p_{3} F(\theta) d \theta \wedge d \phi .
\end{aligned}
$$

We have introduced a parameter $\kappa$ with $\kappa=1,-1$ for $\Sigma^{2}$ being $S^{2}$ or $H^{2}$, respectively. We also note that $F^{\prime \prime}(\theta)=$ $-\kappa F(\theta)$.

With the $S O(2) \times S O(2) \times S O(2)$ singlet scalars given by Eq. (62), we find nonvanishing components of the composite connection:

$$
Q_{A}{ }^{B}=2 g i \sigma_{2} \otimes\left(\begin{array}{lll}
A^{12} & & \\
& A^{34} & \\
& & A^{56}
\end{array}\right) .
$$

With the component $Q_{\hat{\phi} A}{ }^{B}$, the spin connection $\omega^{\hat{\theta} \hat{\phi}}$ can be canceled by imposing the following projector:

$$
\gamma_{\hat{\theta} \hat{\phi}} \epsilon_{A}=\left(i \sigma_{2} \otimes \mathbb{I}_{3}\right)_{A}{ }^{B} \epsilon_{B}
$$

and the twist conditions

$$
2 g p_{1}=2 g p_{2}=2 g p_{3}=1 .
$$

These conditions imply that $p_{1}=p_{2}=p_{3}$. The twist is then obtained from the diagonal subgroup $S O(2)_{\text {diag }} \subset$ $S O(2) \times S O(2) \times S O(2)$ as in the pure $N=4$ and $N=5$ gauged supergravities studied in Refs. [78,51], respectively. For consistency, we will also set $A_{3}=A_{2}=A_{1}$.

For all $p_{i}$ nonvanishing, the twists allow all the supersymmetries corresponding to $\epsilon^{A}, A=1,2, \ldots, 6$, to be unbroken subject to the projector (133). We also note some useful relations for deriving the full set of BPS equations. Using $\epsilon_{\hat{0} \hat{r} \hat{\theta} \hat{\phi}}=1$ and $\gamma_{5} \epsilon_{A}=-\epsilon_{A}$, we find

$$
\gamma^{\hat{0} \hat{r}} \epsilon_{A}=-i \gamma^{\hat{\theta} \hat{\phi}} \epsilon_{A}=\left(\sigma_{2} \otimes \mathbb{I}_{3}\right)_{A}{ }^{B} \epsilon_{B} .
$$

It turns out that we need to turn on the $S O(2) \sim U(1)$ gauge field of $U(6) \sim S U(6) \times U(1)$ in order to find a consistent set of BPS equations. We similarly take the ansatz for this $U(1)$ gauge field to be

$$
\begin{aligned}
& A^{0}=A_{0}(r) d t-p_{0} F^{\prime}(\theta) d \phi \quad \text { and } \\
& F^{0}=A_{0}^{\prime}(r) d r \wedge d t+\kappa p_{0} d \theta \wedge d \phi .
\end{aligned}
$$


We also note that both $A^{0}$ and $A^{I J}$ can appear in the BPS equations due to the off-diagonal element of the scalar coset representative. In particular, we have the relations

$$
\begin{array}{r}
\hat{F}_{A B}^{+}=h_{\Lambda A B} F^{+\Lambda}=h_{0 A B} F^{+0}+\frac{1}{2} h_{I J, A B} F^{+I J}, \\
\hat{F}^{+}=h_{\Lambda 0} F^{+\Lambda}=h_{00} F^{+0}+\frac{1}{2} h_{I J 0} F^{+I J} .
\end{array}
$$

However, $A^{0}$ does not participate in the twist, since $\epsilon_{A}$ are not charged under the $U(1)$ factor outside $S U(6)$.

It is also useful to define the "central charge" matrix

$$
\mathcal{Z}_{A B}=-\frac{1}{\sqrt{2}}\left(\hat{F}_{\hat{\theta} \hat{\phi} A C}^{+}-i \hat{F}_{\hat{0} \hat{r} A C}^{+}\right)\left(i \sigma_{2} \otimes \mathbb{I}_{3}\right)^{C}{ }_{B} .
$$

In the present case, it turns out that $\mathcal{Z}_{A B}$ is proportional to the identity matrix: $\mathcal{Z}_{A B}=\mathcal{Z} \delta_{A B}$.

With all these and the projector (38), we find the following BPS equation, from $\delta \psi_{\hat{\theta} A}$ and $\delta \psi_{\hat{\phi} A}$ :

$$
h^{\prime} e^{i \Lambda}-\mathcal{W}-\mathcal{Z}=0,
$$

which leads to

$$
h^{\prime}= \pm|\mathcal{W}+\mathcal{Z}| \quad \text { and } \quad e^{i \Lambda}= \pm \frac{\mathcal{W}+\mathcal{Z}}{|\mathcal{W}+\mathcal{Z}|}
$$

With the projectors (38) and (135), the condition $\delta \psi_{\hat{0} A}=$ 0 gives

$$
\left(f^{\prime}+2 i g A_{1}\right) e^{i \Lambda}-\mathcal{W}+\mathcal{Z}=0,
$$

which implies

$$
f^{\prime}=\operatorname{Re}\left[e^{-i \Lambda}(\mathcal{W}-\mathcal{Z})\right] \quad \text { and } \quad 2 g A_{1}=\operatorname{Im}\left[e^{-i \Lambda}(\mathcal{W}-\mathcal{Z})\right]
$$

The latter fixes the time component of the gauge fields. Finally, as in the case of domain walls and Janus solutions, the condition $\delta \psi_{\hat{r} A}=0$ determines the $r$ dependence of the Killing spinors giving rise to $\epsilon_{A}=e^{f / 2} \epsilon_{A(0)}$.

Similar to the RG flow case, it turns out that we need to set $\zeta_{1}=\zeta_{2}=\zeta_{3}=0$ for consistency. This gives real $\mathcal{W}$ and $\mathcal{Z}$ resulting in $e^{i \Lambda}= \pm 1$ and $A_{1}=0$. We will also set $A_{0}(r)=0$ for simplicity, although it is not constrained by the previously obtained conditions. In addition, the compatibility between the BPS equations coming from $\delta \chi_{A}$ and $\delta \chi_{A B C}$ requires

$$
p_{0}=\kappa p_{1}
$$

We also note that, in this case, the choice $p_{0}=0$ breaks all supersymmetry. This implies that the $S O(2) \times S O(2) \times$ $S O(2)$ twist needs to be accompanied by the $U(1)$ gauge field $A_{\mu}^{0}$.

With all these, we find a consistent set of BPS equations given by

$$
\begin{aligned}
\varphi_{1}^{\prime} & =-\frac{\partial|\mathcal{W}+\mathcal{Z}|}{\partial \varphi_{1}} \\
& =\frac{1}{2} e^{-\varphi_{1}-\varphi_{2}-\varphi_{3}}\left[2 g\left(1+e^{2\left(\varphi_{2}+\varphi_{3}\right)}-e^{2\left(\varphi_{1}+\varphi_{2}\right)}-e^{2\left(\varphi_{1}+\varphi_{3}\right)}\right)-p_{1} \kappa e^{-2 h+2 \varphi_{1}+2 \varphi_{2}+\varphi_{3}}\right], \\
\varphi_{2}^{\prime} & =-\frac{\partial|\mathcal{W}+\mathcal{Z}|}{\partial \varphi_{2}} \\
& =\frac{1}{2} e^{-\varphi_{1}-\varphi_{2}-\varphi_{3}}\left[2 g\left(1-e^{2\left(\varphi_{2}+\varphi_{3}\right)}-e^{2\left(\varphi_{1}+\varphi_{2}\right)}+e^{2\left(\varphi_{1}+\varphi_{3}\right)}\right)-p_{1} \kappa e^{-2 h+2 \varphi_{1}+2 \varphi_{2}+\varphi_{3}}\right], \\
\varphi_{3}^{\prime} & =-\frac{\partial|\mathcal{W}+\mathcal{Z}|}{\partial \varphi_{3}} \\
& =\frac{1}{2} e^{-\varphi_{1}-\varphi_{2}-\varphi_{3}}\left[2 g\left(1-e^{2\left(\varphi_{2}+\varphi_{3}\right)}+e^{2\left(\varphi_{1}+\varphi_{2}\right)}-e^{2\left(\varphi_{1}+\varphi_{3}\right)}\right)-p_{1} \kappa e^{-2 h+2 \varphi_{1}+2 \varphi_{2}+\varphi_{3}}\right], \\
h^{\prime} & =|\mathcal{W}+\mathcal{Z}| \\
& =\frac{1}{2} e^{-\varphi_{1}-\varphi_{2}-\varphi_{3}}\left[2 g\left(1+e^{2\left(\varphi_{2}+\varphi_{3}\right)}+e^{2\left(\varphi_{1}+\varphi_{2}\right)}+e^{2\left(\varphi_{1}+\varphi_{3}\right)}\right)+p_{1} \kappa e^{-2 h+2 \varphi_{1}+2 \varphi_{2}+\varphi_{3}}\right],
\end{aligned}
$$




$$
\begin{aligned}
f^{\prime} & =|\mathcal{W}-\mathcal{Z}| \\
& =\frac{1}{2} e^{-\varphi_{1}-\varphi_{2}-\varphi_{3}}\left[2 g\left(1+e^{2\left(\varphi_{2}+\varphi_{3}\right)}+e^{2\left(\varphi_{1}+\varphi_{2}\right)}+e^{2\left(\varphi_{1}+\varphi_{3}\right)}\right)-p_{1} \kappa e^{-2 h+2 \varphi_{1}+2 \varphi_{2}+\varphi_{3}}\right] .
\end{aligned}
$$

For an $\mathrm{AdS}_{2} \times \Sigma^{2}$ fixed point to exist, we require that $\varphi_{1}^{\prime}=$ $\varphi_{2}^{\prime}=\varphi_{3}^{\prime}=h^{\prime}=0$ and $f^{\prime} \sim \frac{1}{L_{\mathrm{AdS}}}$. It can be easily verified that the above equations do not admit any $\mathrm{AdS}_{2} \times \Sigma^{2}$ fixed points.

Although there is no supersymmetric $\operatorname{AdS}_{2} \times \Sigma^{2}$ fixed point, we are able to analytically obtain the complete solution to these BPS equations. Since it might be useful for some holographic studies, we will present the solution here. By changing to a new radial coordinate $\rho$ using the relation $\frac{d \rho}{d r}=e^{\varphi_{3}}$, we can form the following linear combinations:

$$
\frac{d}{d \rho}\left(\varphi_{1}-\varphi_{2}\right)=2 g\left(e^{\varphi_{2}-\varphi_{1}}-e^{\varphi_{1}-\varphi_{2}}\right)
$$

and

$$
\frac{d}{d \rho}\left(\varphi_{2}-\varphi_{3}\right)=2 g\left(e^{\varphi_{1}-\varphi_{2}}-e^{\varphi_{1}+\varphi_{2}-2 \varphi_{3}}\right) .
$$

The first equation can be solved by

$$
\varphi_{1}=\ln \left[\frac{e^{\varphi_{2}}\left(e^{4 g \rho}+e^{4 g \rho_{0}}\right)}{e^{4 g \rho}-e^{4 g \rho_{0}}}\right]
$$

with an integration constant $\rho_{0}$. Using this result in the second equation, we find the solution

$$
\varphi_{2}=\ln \left[\frac{e^{\varphi_{3}-2 g \rho}\left(e^{4 g \rho}-e^{4 g \rho_{0}}\right)}{\sqrt{e^{4 g \rho}+e^{8 g \rho_{0}}+8 g C}}\right]
$$

with another integration constant $C$.

By treating $f$ and $h$ as functions of $\varphi_{3}$, we find the following solutions for $f$ and $h$ :

$$
\begin{aligned}
f= & -\frac{1}{2} \ln \left[e^{4 g \rho_{0}}\left(256 g^{3} C \tilde{C}-16 g \tilde{C} e^{8 g \rho_{0}}+\kappa p_{1} \ln \left[\frac{1+e^{8 g\left(\rho-\rho_{0}\right)}+8 g C e^{4 g \rho-8 g \rho_{0}}}{e^{8 g\left(\rho-\rho_{0}\right)}-1}\right]\right)\right. \\
& \left.-8 g C \kappa p_{1} \tanh ^{-1} e^{4 g\left(\rho-\rho_{0}\right)}\right]+h, \\
h= & \frac{1}{2} \ln \left[e^{4 g \rho_{0}}\left(16 g \tilde{C}\left(e^{8 g \rho_{0}}-16 g^{2} C^{2}\right)-\kappa p_{1} \ln \left[\frac{1+e^{8 g\left(\rho-\rho_{0}\right)}+8 g C e^{4 g \rho-8 g \rho_{0}}}{e^{8 g\left(\rho-\rho_{0}\right)}-1}\right]\right)\right. \\
& \left.+8 g C \kappa p_{1} \tanh ^{-1} e^{4 g\left(\rho-\rho_{0}\right)}\right]+\frac{1}{2} \ln \left[\frac{e^{12 g \rho_{0}}\left(1-e^{8 g\left(\rho-\rho_{0}\right)}\right)^{2}}{8 g\left(e^{8 g \rho_{0}}-16 g^{2} C^{2}\right)}\right]+\varphi_{3}-4 g \rho .
\end{aligned}
$$

Finally, the solution $\varphi_{3}(\rho)$ can be given implicitly in the following equation:

$$
4 C_{0} e^{4 g \rho}\left(e^{8 g \rho_{0}}+e^{8 g \rho}+8 g C e^{4 g \rho}\right)=\beta_{0}+\beta_{1} \ln \left[\frac{e^{4 g\left(\rho_{0}-\rho\right)}+1}{e^{4 g\left(\rho_{0}-\rho\right)}-1}\right]+\beta_{2} \ln \left[\frac{e^{8 g\left(\rho-\rho_{0}\right)}-1}{1+e^{8 g\left(\rho-\rho_{0}\right)}+8 g C e^{4 g \rho}}\right]
$$

in which $C_{0}$ is a constant and the coefficients $\beta_{0}, \beta_{1}$, and $\beta_{2}$ are defined, respectively, by

$$
\begin{gathered}
\beta_{0}=-16 g \tilde{C} e^{4 \rho_{0}}\left(16 C g^{2}-e^{8 g \rho_{0}}\right)\left[2 e^{4 \varphi_{3}+8 g\left(\rho+\rho_{0}\right)}+8 g C e^{4 g \rho}\left(e^{8 g \rho}+e^{8 g \rho_{0}}\right)+\left(e^{16 g \rho_{0}}+e^{16 g \rho}\right)\left(1-e^{4 \varphi_{3}}\right)+2 e^{8 g\left(\rho+\rho_{0}\right)}\right], \\
\beta_{1}=\left[\frac{\kappa p_{1}}{2\left(e^{4 g \rho}+e^{4 g\left(3 \rho-2 \rho_{0}\right)}+8 C g e^{8 g\left(\rho-\rho_{0}\right)}\right)}\right]\left[e^{12 g \rho}+e^{4 g\left(\rho+2 \rho_{0}\right)}+4 C g e^{8 g \rho}\left(3+e^{4 \varphi_{3}}\right)\right. \\
\left.+16 g^{2} C^{2} e^{4 g \rho}\left(1+e^{8 g\left(\rho-\rho_{0}\right)}\right)-2 C g\left(e^{4 \varphi_{3}}-1\right)\left(e^{8 g \rho_{0}}+e^{8 g\left(2 \rho-\rho_{0}\right)}\right)\right], \\
\beta_{2}=\frac{\kappa p_{1}\left[\left(e^{8 g \rho_{0}}+e^{8 g \rho}+8 C g e^{4 g \rho}\right)^{2}-e^{4 \rho_{3}}\left(e^{8 g \rho_{0}}-e^{8 g \rho}\right)^{2}\right]}{4 e^{4 g\left(\rho-\rho_{0}\right)}\left(e^{8 g \rho_{0}}+e^{8 g \rho}+8 C g e^{4 g \rho}\right)} .
\end{gathered}
$$


Since there is no $\mathrm{AdS}_{2} \times \Sigma^{2}$ fixed point in the IR, the solution describes a flow from the locally supersymmetric $\mathrm{AdS}_{4}$ vacuum to a curved domain wall with world volume $\mathbb{R} \times \Sigma^{2}$. According to the AdS/CFT correspondence, the solution is expected to describe an RG flow from the $N=6$ SCFT in three dimensions to a supersymmetric quantum mechanics in the IR. The latter arises from the former by a twisted compactification on $\Sigma^{2}$.

\section{B. $S O(2) \times S O(4)$ symmetric solutions}

We now look at a truncation of the previous result by setting $p_{2}=p_{3}=0$ and $\varphi_{2}=\varphi_{3}=0$. The resulting solutions will preserve $S O(2) \times S O(4)$ symmetry with the twist performed along the $S O(2)$ factor. In this case, the supersymmetry corresponding to $\epsilon^{3,4,5,6}$ will be broken, since we cannot perform a twist along these directions. With $\epsilon^{3,4,5,6}=0$, we find the BPS equations

$\varphi^{\prime}=\frac{1}{4} e^{-2 h-\varphi}\left[8 g e^{2 h}-p_{0}+\kappa p_{1}-e^{2 \varphi}\left(8 g e^{2 h}+p_{0}+\kappa p_{1}\right)\right]$,

$h^{\prime}=\frac{1}{4} e^{-2 h-\varphi}\left[8 g e^{2 h}-p_{0}+\kappa p_{1}+e^{2 \varphi}\left(8 g e^{2 h}+p_{0}+\kappa p_{1}\right)\right]$,

$f^{\prime}=\frac{1}{4} e^{-2 h-\varphi}\left[8 g e^{2 h}+p_{0}-\kappa p_{1}+e^{2 \varphi}\left(8 g e^{2 h}-p_{0}-\kappa p_{1}\right)\right]$,

in which we have set $\varphi_{1}=\varphi$. We also note that, with only the $S O(2)$ twist, it is not necessary to set $p_{0}=\kappa p_{1}$. However, the existence of an $\mathrm{AdS}_{2} \times \Sigma^{2}$ fixed point requires vanishing $p_{0}$. For $p_{0}=0$, we find a fixed point

$$
\varphi=\varphi_{0}, \quad h=\frac{1}{2} \ln \left[-\frac{\kappa p_{1}}{8 g}\right], \quad L_{\mathrm{AdS}_{2}}=\frac{1}{8 g \cosh 2 \varphi_{0}} .
$$

for constant $\varphi_{0}$. This is an $\mathrm{AdS}_{2} \times H^{2}$ fixed point, since the reality of $h$ implies $\kappa=-1$.

The complete flow solution can be obtained by using the same procedure as in the previous sections. The resulting solution is given by

$$
\begin{gathered}
h=\varphi-\ln \left(1-e^{2 \varphi}\right)+C, \\
f=h-2 \varphi+\ln \left[\kappa p_{1}\left(1+e^{4 \varphi}\right)+2 e^{2 \varphi}\left(4 g-\kappa p_{1}\right)\right],
\end{gathered}
$$

$$
\begin{aligned}
8 g\left(\rho-\rho_{0}\right)= & 2 \sqrt{\frac{2 g}{\kappa p_{1}-2 g}} \tan ^{-1}\left[\frac{4 g+\kappa p_{1}\left(e^{2 \varphi}-1\right)}{2 \sqrt{2 g\left(\kappa p_{1}-2 g\right)}}\right] \\
& +\ln \left[\frac{\kappa p_{1}\left(1+e^{4 \varphi}\right)+2 e^{2 \varphi}\left(4 g-\kappa p_{1}\right)}{\left(1-e^{2 \varphi}\right)^{2}}\right]
\end{aligned}
$$

in which we have defined the new radial coordinate $\rho$ by $\frac{d \rho}{d r}=e^{\varphi}$. We have neglected the integration constant of $f$ by absorbing it in the rescaling of the time coordinate $t$.

Near $r \sim \rho \rightarrow \infty$, we find

$$
\varphi \sim e^{-4 g r}, \quad h \sim f \sim 4 g r
$$

which gives an asymptotically locally $\mathrm{AdS}_{4}$ critical point. On the other hand, by choosing $\varphi_{0}=\frac{1}{2} \ln \left(1-2 \sqrt{-\frac{2 g}{\kappa p_{1}}}\right)$ and $C=-\varphi_{0}$, we find that as $\varphi \rightarrow \varphi_{0}$

$h \sim \frac{1}{2} \ln \left[-\frac{\kappa p_{1}}{8 g}\right] \quad$ and $\quad f \sim 8 g r \frac{1-\sqrt{-\frac{2 g}{\kappa p_{1}}}}{\sqrt{1-2 \sqrt{-\frac{2 g}{\kappa p_{1}}}}}$,

which is the $\mathrm{AdS}_{2} \times H^{2}$ fixed point identified above.

It should be noted that, in this case, the solution can be regarded as a solution of a truncated $N=2$ gauged supergravity. In particular, the solution with vanishing scalar corresponds to a universal RG flow across dimension of which the uplifts to $\mathrm{M}$ theory and massive type IIA theory have been extensively studied in Refs. [67].

\section{C. $U(3)$ symmetric solutions}

As a final case, we consider $U(3)$ symmetric solutions with a twist performed along the $S O(2) \sim U(1)$ factor. The corresponding gauge generator of this $U(1)$ factor is given by $X_{14}+X_{25}+X_{36}$. We then turn on the following gauge fields:

$$
\mathcal{A}=A^{14}=A^{25}=A^{36}=A(r) d t-\kappa p F^{\prime}(\theta) d \phi .
$$

With the $U(3)$ singlet scalar given in Eq. (51), we find the composite connection

$$
Q_{A}{ }^{B}=2 g i \mathcal{A}\left(\mathbb{I}_{3} \otimes \sigma_{2}\right)_{A}{ }^{B} .
$$

The twist is implemented by imposing

$$
\gamma_{\hat{\theta} \hat{\phi}} \epsilon_{A}=\left(\mathbb{I}_{3} \otimes i \sigma_{2}\right)_{A}{ }^{B} \epsilon_{B} \quad \text { and } \quad 2 g p=1 .
$$

We also note that, similar to the $S O(2) \times S O(2) \times S O(2)$ twist, all $\epsilon_{A}$ can be nonvanishing. In addition, we also need nonvanishing $A^{0}$, which we will again use the ansatz (136). In this case, consistency requires $p_{0}=-\kappa p$. 
As in the RG flow case, we need to set $\zeta=0$ for consistency between the BPS equations and the field equations. This again results in $A(r)=0$. Repeating the same analysis as in the previous cases, we find the following BPS equations:

$$
\begin{aligned}
\varphi^{\prime} & =g\left(e^{-\varphi}-e^{3 \varphi}\right)+\frac{1}{2} \kappa p e^{-2 h-3 \varphi}, \\
h^{\prime} & =g\left(3 e^{-\varphi}+e^{3 \varphi}\right)+\frac{1}{2} \kappa p e^{-2 h-3 \varphi}, \\
\varphi^{\prime} & =g\left(3 e^{-\varphi}+e^{3 \varphi}\right)-\frac{1}{2} \kappa p e^{-2 h-3 \varphi} .
\end{aligned}
$$

It is easily verified that there is no $\mathrm{AdS}_{2} \times \Sigma^{2}$ fixed point in these equations. In this case, we are not able to obtain the analytic flow solution.

\section{CONCLUSIONS AND DISCUSSIONS}

In this paper, we have studied $N=6$ gauged supergravity in four dimensions with the $S O(6)$ gauge group. The gauged supergravity can be obtained from a truncation of the maximal $N=8$ theory with the $S O(8)$ gauge group. There is a unique $N=6$ supersymmetric $\mathrm{AdS}_{4}$ vacuum preserving the full $S O(6)$ gauge symmetry. This can be identified with $\mathrm{AdS}_{4} \times C P^{3}$ geometry in type IIA theory dual to an $N=6$ SCFT in three dimensions. We have found a number of RG flow solutions with various symmetries from this $N=6$ SCFT to possible nonconformal phases in the IR. In particular, there is one solution, breaking the $S O(6)$ R symmetry to $S O(2) \times S O(4)$, with unbroken $N=6$ Poincaré supersymmetry. This is precisely in agreement with the field theory result on mass deformations of $N=6$ SCFTs given in Ref. [25]. Other solutions preserve $U(3), S O(3)$, and $S O(2) \times S O(2) \times S O(2)$ symmetries. While most of the solutions preserve $N=6$ supersymmetry, in the case of $S O(3)$ symmetry, it is possible to find $N=2$ supersymmetric solutions. We have analytically given all of these solutions and also checked that, except for the $N=2$ solution, the resulting IR singularities are physical by the criterion given in Ref. [77].

We have also considered more complicated solutions by generalizing the flat domain walls to the curved ones. In the case of $\mathrm{AdS}_{3}$-sliced domain walls, we have found a supersymmetric Janus solution, describing a two-dimensional conformal defect within the $N=6$ SCFT, with $S O(2) \times S O(4)$ symmetry and $N=(2,4)$ supersymmetry on the defect. The resulting solution takes the same form as those given in $N=8, N=5$, and $N=3$ gauged supergravities studied in Refs. [52,51,53], respectively. We therefore argue that these solutions are related to the $N=8$ solution by truncations. In order for Janus solutions to exist, it is necessary that pseudoscalars are nonvanishing as pointed out in Ref. [52]. It turns out that, among the remaining cases studied in this work, only the $S O(3)$ invariant sector could possibly admit supersymmetric Janus solutions.

Furthermore, we have studied supersymmetric solutions of the form $\mathrm{AdS}_{2} \times \Sigma^{2}$ and the interpolating solutions between these geometries and the $N=6 \mathrm{AdS}_{4}$ vacuum. We have found one $\mathrm{AdS}_{2} \times H^{2}$ fixed point with $S O(2) \times$ $S O(4)$ symmetry from $S O(2)$ twist. The solution interpolating between this fixed point and the $\mathrm{AdS}_{4}$ vacuum preserves two supercharges, while the IR fixed point $\mathrm{AdS}_{2} \times H^{2}$ has four supercharges. Holographically, this solution corresponds to an RG flow from the $N=6$ SCFT to superconformal quantum mechanics which is useful in computing black hole entropy along the lines of Refs. [67-69].

For $S O(2) \times S O(2) \times S O(2)$ twist, the BPS equations are more complicated but admit no $\mathrm{AdS}_{2} \times \Sigma^{2}$ fixed point. However, in this case, we are able to obtain a complete flow solution between the $\mathrm{AdS}_{4}$ critical point to a curved domain wall with world volume $\mathbb{R} \times \Sigma^{2}$ in the IR. The solution preserves $N=6$ supersymmetry in three dimensions, or 12 supercharges, and $S O(2) \times S O(2) \times S O(2)$ symmetry. This should be dual to a twisted compactification on $\Sigma^{2}$ of the UV $N=6$ SCFT to a supersymmetric quantum mechanics in the IR. We have also looked for $\mathrm{AdS}_{2} \times \Sigma^{2}$ geometries from an $S O(2) \sim U(1)$ twist in the case of $U(3)$ symmetric solutions, but there do not exist any $\mathrm{AdS}_{2} \times \Sigma^{2}$ fixed points.

Since all the solutions presented here are fully analytic, we hope they could be useful in the study of gauge and gravity holography and other related aspects. We also note that most of the structures of the solutions are very similar to those of the $N=5$ gauged supergravity studied in Ref. [51]. In particular, the $N=6$ Poincaré supersymmetry in three dimensions is unbroken on the domain wall solutions if there are no nonvanishing pseudoscalars. Unlike in the $N=5$ theory, we are not able to find a definite conclusion on whether this is true, in general, due to a more complicated scalar coset manifold. However, many similarities in the structures of various types of supersymmetric solutions suggest that this should be the case.

There are a number of directions to extend the present work, which is clearly only the first step in classifying supersymmetric solutions of $N=6$ gauged supergravity. First of all, it would be interesting to uplift the RG flow solutions to M theory via the embedding in $N=8$ gauged supergravity, which, in turn, can be obtained from a truncation of M theory on $S^{7}$. The time component $g_{00}$ of the 11-dimensional metric can be used to determine whether the four-dimensional singularities, in particular, the $N=2$ case, are physically acceptable in M theory using the criterion given in Ref. [79]. This would lead to a complete holographic description of mass deformations of $N=6 \mathrm{CSM}$ theory and possible related M-brane configurations. 
We have considered only gauged supergravity with the $S O(6)$ gauge group electrically embedded in the global $S O^{*}(12)$ symmetry. It would be interesting to study magnetic and dyonic gaugings involving also magnetic gauge fields. In particular, performing a similar study in the case of $N=6$ gauged supergravity with the electric-magnetic phase $\omega$ (see [80-82]) could be of particular interest, since, in the omega deformed $N=8$ theory, the structure of vacua and domain walls are much richer than the electric counterpart; see [43,83-85] for more detail. In addition, the study of genuine $N=6$ gaugings which cannot be embedded in the $N=8$ theory is worth considering. In this case, the gaugings do not satisfy extra quadratic constraints coming from the truncation of the $N=8$ theory (see the discussion in Ref. [86]), so the corresponding solutions cannot be embedded in the maximal theory.

\section{ACKNOWLEDGMENTS}

We give special thanks to $\mathrm{M}$. Trigiante for valuable correspondence. We also thank C. Panwisawas for his help in finding some literature. This work is supported by The Thailand Research Fund (TRF) under Grant No. RSA6280022.
[1] J. M. Maldacena, The large $N$ limit of superconformal field theories and supergravity, Adv. Theor. Math. Phys. 2, 231 (1998).

[2] S. S. Gubser, I. R. Klebanov, and A. M. Polyakov, Gauge theory correlators from non-critical string theory, Phys. Lett. B 428, 105 (1998).

[3] E. Witten, Anti De Sitter space and holography, Adv. Theor. Math. Phys. 2, 253 (1998).

[4] P. Meessen, T. Ortin, and S. Vaula, All the timelike supersymmetric solutions of all ungauged $d=4$ supergravities, J. High Energy Phys. 11 (2010) 072.

[5] L. Andrianopoli, R. D’Auria, S. Ferrara, P. A. Grassi, and M. Trigiante, Exceptional $N=6$ and $N=2 \mathrm{AdS}_{4}$ supergravity, and zero-center modules, J. High Energy Phys. 04 (2009) 074.

[6] B. de Wit, H. Samtleben, and M. Trigiante, The maximal $D=4$ supergravities, J. High Energy Phys. 06 (2007) 049.

[7] B. de Wit and H. Nicolai, $N=8$ Supergravity, Nucl. Phys. B208, 323 (1982).

[8] B. de Wit, H. Samtleben, and M. Trigiante, On Lagrangians and gaugings of maximal supergravities, Nucl. Phys. B655, 93 (2003).

[9] B. de Wit, H. Samtleben, and M. Trigiante, Magnetic charges in local field theory, J. High Energy Phys. 09 (2005) 016.

[10] S. Lust, P. Ruter, and J. Louis, Maximally supersymmetric AdS solutions and their moduli spaces, J. High Energy Phys. 03 (2018) 019.

[11] B. E. W. Nilsson and C. N. Pope, Hopf fibration of elevendimensional supergravity, Classical Quantum Gravity 1, 499 (1984).

[12] G. Arutyunov and S. Frolov, Superstrings on $\mathrm{AdS}_{4} \times C P^{3}$ as a coset sigma-model', J. High Energy Phys. 09 (2008) 129.

[13] B. Stefanski, Jr., Green-Schwarz action for Type IIA strings on $\mathrm{AdS}_{4} \times C P^{3}$, Nucl. Phys. B808, 80 (2009).

[14] R. D Auria, P. Fre, P. A. Grassi, and M. Trigiante, Superstrings on $\mathrm{AdS}_{4} \times C P^{3}$ from supergravity, Phys. Rev. D 79, 086001 (2009).
[15] G. Bonelli, P. A. Grassi, and H. Safaai, Exploring pure spinor string theory on $\mathrm{AdS}_{4} \times C P^{3}$, J. High Energy Phys. 10 (2008) 085.

[16] O. Aharony, O. Bergman, D. L. Jafferis, and J. Maldacena, $N=6$ superconformal Chern-Simons-matter theories, M2-branes and their gravity duals, J. High Energy Phys. 10 (2008) 091.

[17] I. Bena, The M-theory dual of a 3 dimensional theory with reduced supersymmetry, Phys. Rev. D 62, 126006 (2000).

[18] J. Bagger and N. Lambert, Modeling multiple M2s, Phys. Rev. D 75, 045020 (2007).

[19] J. Bagger and N. Lambert, Gauge symmetry and supersymmetry of multiple M2-branes, Phys. Rev. D 77, 065008 (2008).

[20] J. Bagger and N. Lambert, Comments on multiple M2-branes, J. High Energy Phys. 1 (2008) 105.

[21] A. Gustavsson, Algebraic structures on parallel M2-branes, Nucl. Phys. B811, 66 (2009).

[22] A. Basu and J. A. Harvey, The M2-M5 brane system and a generalized Nahms equation, Nucl. Phys. B713, 136 (2005).

[23] J. H. Schwarz, Superconformal Chern-Simons theories, J. High Energy Phys. 11 (2004) 078.

[24] O. Aharony, O. Bergman, and D. L. Jafferis, Fractional M2-branes, J. High Energy Phys. 11 (2008) 043.

[25] K. Hosomichi, K. M. Lee, S. Lee, S. Lee, and J. Park, $N=5,6$ Superconformal Chern-Simons Theories and M2branes on Orbifolds, J. High Energy Phys. 09 (2008) 002.

[26] M. Schnabl and Y. Tachikawa, Classification of $N=6$ superconformal theories of ABJM type, J. High Energy Phys. 09 (2010) 103.

[27] J. Bagger and N. Lambert, Three-algebras and $N=6$ Chern-Simons gauge theories, Phys. Rev. D 79, 025002 (2009).

[28] N. Lambert and C. Papageorgakis, Relating $U(N) \times U(N)$ to $S U(N) \times S U(N)$ Chern-Simons membrane theories, J. High Energy Phys. 04 (2010) 104.

[29] P. de Medeiros, J. Figueroa-OFarrill, E. Mendez-Escobar, and P. Ritter, On the Lie-algebraic origin of metric 3-algebras, Commun. Math. Phys. 290, 871 (2009). 
[30] E. A. Bergshoeff, O. Hohm, D. Roest, H. Samtleben, and E. Sezgin, The superconformal gaugings in three dimensions, J. High Energy Phys. 09 (2008) 101.

[31] F. M. Chen, Symplectic Three-algebra unifying $N=5,6$ superconformal Chern-Simons-matter theories, J. High Energy Phys. 08 (2010) 077.

[32] E. A. Bergshoeff, M. de Roo, and O. Hohm, Multiple M2-branes and the embedding tensor, Classical Quantum Gravity 25, 142001 (2008).

[33] E. A. Bergshoeff, M. de Roo, O. Hohm, and D. Roest, Multiple membranes from gauged supergravity, J. High Energy Phys. 08 (2008) 091.

[34] P. de Medeiros, J. Figueroa-OFarrill, and E. MendezEscobar, Superpotentials for superconformal Chern-Simons theories from representation theory, J. Phys. A 42, 485204 (2009).

[35] J. Bagger and G. Bruhn, Three-algebras in $N=5,6$ superconformal Chern-Simons theories: Representations and relations, Phys. Rev. D 83, 025003 (2011).

[36] M. Honda, Exact relations between M2-brane theories with and without orientifolds, J. High Energy Phys. 06 (2016) 123.

[37] R. Corrado, K. Pilch, and N. P. Warner, An $N=2$ supersymmetric membrane flow, Nucl. Phys. B629, 74 (2002).

[38] C. N. Gowdigere and N. P. Warner, Flowing with eight supersymmetries in M-theory and F-theory, J. High Energy Phys. 12 (2003) 048.

[39] K. Pilch, A. Tyukov, and N. P. Warner, Flowing to higher dimensions: A new strongly-coupled phase on M2 branes, J. High Energy Phys. 11 (2015) 170.

[40] C. Ahn and K. Woo, Supersymmetric domain wall and RG flow from 4-dimensional gauged $N=8$ Supergravity, Nucl. Phys. B599, 83 (2001).

[41] C. Ahn and T. Itoh, An $N=1$ Supersymmetric $G_{2}$-invariant Flow in M-theory, Nucl. Phys. B627, 45 (2002).

[42] N. Bobev, N. Halmagyi, K. Pilch, and N. P. Warner, Holographic, $N=1$ Supersymmetric RG flows on M2 branes, J. High Energy Phys. 09 (2009) 043.

[43] A. Guarino, On new maximal supergravity and its BPS domain-walls, J. High Energy Phys. 02 (2014) 026.

[44] J. Tarrio and O. Varela, Electric/magnetic duality and RG flows in $\mathrm{AdS}_{4} / \mathrm{CFT}_{3}$, J. High Energy Phys. 01 (2014) 071.

[45] Y. Pang, C. N. Pope, and J. Rong, Holographic RG flow in a new $S O(3) \times S O(3)$ sector of $\omega$-deformed $S O(8)$ gauged $N=8$ supergravity, J. High Energy Phys. 08 (2015) 122.

[46] P. Karndumri, Holographic RG flows in $N=3$ ChernSimons-Matter theory from $N=34$ D gauged supergravity, Phys. Rev. D 94, 045006 (2016).

[47] P. Karndumri and K. Upathambhakul, Gaugings of fourdimensional $N=3$ supergravity and $\mathrm{AdS}_{4} / \mathrm{CFT}_{3}$ holography, Phys. Rev. D 93, 125017 (2016).

[48] P. Karndumri, Supersymmetric deformations of 3D SCFTs from tri-sasakian truncation, Eur. Phys. J. C 77, 130 (2017).

[49] P. Karndumri and K. Upathambhakul, Supersymmetric RG flows and Janus from type II orbifold compactification, Eur. Phys. J. C 77, 455 (2017).

[50] P. Karndumri and K. Upathambhakul, Holographic RG flows in $N=4$ SCFTs from half-maximal gauged supergravity, Eur. Phys. J. C 78, 626 (2018).
[51] P. Karndumri and C. Maneerat, Supersymmetric solutions from $N=5$ gauged supergravity, Phys. Rev. D 101, 126015 (2020).

[52] N. Bobev, K. Pilchand, and N. P. Warner, Supersymmetric Janus solutions in four dimensions, J. High Energy Phys. 06 (2014) 058.

[53] P. Karndumri, Supersymmetric Janus solutions in fourdimensional $N=3$ gauged supergravity, Phys. Rev. D 93, 125012 (2016).

[54] M. Suh, Supersymmetric Janus solutions of dyonic ISO(7)gauged $N=8$ supergravity, J. High Energy Phys. 04 (2018) 109.

[55] N. Kim and S. J. Kim, Re-visiting supersymmetric Janus solutions: A perturbative construction, Chin. Phys. C 44, 073104 (2020).

[56] M. Cvetic, M. Duff, P. Hoxha, J. T. Liu, H. Lu et al., Embedding AdS black holes in ten-dimensions and elevendimensions, Nucl. Phys. B558, 96126 (1999).

[57] M. J. Duff and J. T. Liu, Anti-de sitter black holes in gauged $N=8$ supergravity, Nucl. Phys. B554, 237 (1999).

[58] J. P. Gauntlett, N. Kim, S. Pakis, and D. Waldram, Membranes wrapped on holomorphic curves, Phys. Rev. D 65, 026003 (2001).

[59] S. L. Cacciatori and D. Klemm, Supersymmetric AdS(4) black holes and attractors, J. High Energy Phys. 01 (2010) 085.

[60] G. DallAgata and A. Gnecchi, Flow equations and attractors for black holes in $N=2 U(1)$ gauged supergravity, J. High Energy Phys. 03 (2011) 037.

[61] K. Hristov and S. Vandoren, Static supersymmetric black holes in $\mathrm{AdS}_{4}$ with spherical symmetry, J. High Energy Phys. 04 (2011) 047.

[62] N. Halmagyi, BPS black hole horizons in $N=2$ gauged supergravity, J. High Energy Phys. 02 (2014) 051.

[63] N. Halmagyi, M. Petrini, and A. Zaffaroni, BPS black holes in AdS $_{4}$ from M-theory, J. High Energy Phys. 08 (2013) 124.

[64] A. Guarino and J. Tarrio, BPS black holes from massive IIA on $S^{6}$, J. High Energy Phys. 09 (2017) 141.

[65] A. Guarino, BPS black hole horizons from massive IIA, J. High Energy Phys. 08 (2017) 100.

[66] P. Karndumri, Supersymmetric $\mathrm{AdS}_{2} \times \Sigma_{2}$ solutions from tri-sasakian truncation, Eur. Phys. J. C 77, 689 (2017).

[67] F. Azzurli, N. Bobev, P. M. Crichigno, V. S. Min, and A. Zaffaroni, A universal counting of black hole microstates in $\mathrm{AdS}_{4}$, J. High Energy Phys. 02 (2018) 054.

[68] F. Benini, H. Khachatryan, and P. Milan, Black hole entropy in massive type IIA, Classical Quantum Gravity 35, 035004 (2018).

[69] S. M. Hosseini, K. Hristov, and A. Passias, Holographic microstate counting for $\mathrm{AdS}_{4}$ black holes in massive IIA supergravity, J. High Energy Phys. 10 (2017) 190.

[70] B. de Wit and H. Nicolai, The consistency of the $S^{7}$ truncation in $D=11$ supergravity, Nucl. Phys. B281, 211 (1987).

[71] B. de Wit, H. Nicolai, and N. P. Warner, The embedding of gauged $N=8$ supergravity into $d=11$ supergravity, Nucl. Phys. B255, 29 (1985).

[72] H. Nicolai and K. Pilch, Consistent truncation of $d=11$ supergravity on $\mathrm{AdS}_{4} \times S^{7}$, J. High Energy Phys. 03 (2012) 099. 
[73] B. de Wit and H. Nicolai, Deformations of gauged $S O(8)$ supergravity and supergravity in eleven dimensions, J. High Energy Phys. 05 (2013) 077.

[74] H. Godazgar, M. Godazgar, and Hermann Nicolai, Testing the non-linear flux ansatz for maximal supergravity, Phys. Rev. D 87, 085038 (2013).

[75] H. Godazgar, M. Godazgar, O. Krueger, and H. Nicolai, Consistent 4-form fluxes for maximal supergravity, J. High Energy Phys. 10 (2015) 169.

[76] N. P. Warner, Some properties of the scalar potential in gauged supergravity theories, Nucl. Phys. B231, 250 (1984).

[77] S. S. Gubser, Curvature singularities: The good, the bad and the naked, Adv. Theor. Math. Phys. 4, 679 (2000).

[78] N. Bobev and P. M. Crichigno, Universal RG flows across dimensions and holography, J. High Energy Phys. 12 (2017) 065.

[79] J. Maldacena and C. Nunez, Supergravity description of field theories on curved manifolds and a no go theorem, Int. J. Mod. Phys. A 16, 822 (2001).
[80] A. Borghese, Y. Pang, C. N. Pope, and E. Sezgin, Correlation functions in $\omega$-deformed $\mathrm{N}=6$ supergravity, J. High Energy Phys. 02 (2015) 112.

[81] G. DallAgata, G. Inverso, and M. Trigiante, Evidence for a Family of $S O(8)$ Gauged Supergravity Theories, Phys. Rev. Lett. 109, 201301 (2012).

[82] G. Dall' Agata, G. Inverso, and Al, Marrani, Symplectic deformations of gauged maximal supergravity, J. High Energy Phys. 07 (2014) 133.

[83] A. Borghese, G. Dibitetto, A. Guarino, D. Roest, and O. Varela, The $S U(3)$-invariant sector of new maximal supergravity, J. High Energy Phys. 03 (2013) 082.

[84] A. Gallerati, H. Samtleben, and M. Trigiante, The $N>2$ supersymmetric AdS vacua in maximal supergravity, J. High Energy Phys. 12 (2014) 174.

[85] J. Tarrio and O. Varela, Electric/magnetic duality and RG flows in AdS4/CFT3, J. High Energy Phys. 01 (2014) 071.

[86] D. Roest and H. Samtleben, Twin supergravities, Classical Quantum Gravity 26, 155001 (2009). 